\title{
Coherent energetic interfaces accounting for in-plane degradation
}

\author{
Ali Esmaeili · Ali Javili · Paul Steinmann
}

Received: 9 December 2015 / Accepted: 13 October 2016 / Published online: 9 November 2016

(C) Springer Science+Business Media Dordrecht 2016

\begin{abstract}
Interfaces can play a dominant role in the overall response of a body. The importance of interfaces is particularly appreciated at small length scales due to large area to volume ratios. From the mechanical point of view, this scale dependent characteristic can be captured by endowing a coherent interface with its own elastic resistance as proposed by the interface elasticity theory. This theory proves to be an extremely powerful tool to explain size effects and to predict the behavior of nano-materials. To date, interface elasticity theory only accounts for the elastic response of coherent interfaces and obviously lacks an explanation for inelastic interface behavior such as damage or plasticity. The objective of this contribution is to extend interface elasticity theory to account for damage of coherent interfaces. To this end, a thermodynamically consistent interface elasticity theory with damage is proposed. A local damage model for the interface is presented and is extended towards a non-local damage model. The non-linear governing equations and the weak forms
\end{abstract}

A. Esmaeili · P. Steinmann ( $\varangle)$

Chair of Applied Mechanics, University of

Erlangen-Nuremberg, Egerlandstrasse 5, 91058 Erlangen,

Germany

e-mail: paul.steinmann@1tm.uni-erlangen.de

A. Esmaeili

e-mail: ali.esmaeili@1tm.uni-erlangen.de

A. Javili

Department of Mechanical Engineering, Bilkent

University, 06800 Ankara, Turkey

e-mail: ajavili@bilkent.edu.tr thereof are derived. The numerical implementation is carried out using the finite element method and consistent tangents are listed. The computational algorithms are given in detail. Finally, a series of numerical examples is studied to provide further insight into the problem and to carefully elucidate key features of the proposed theory.

Keywords Interface elasticity - Non-local damage · Cohesive zone $\cdot$ Coherent interfaces $\cdot$ Finite element method $\cdot$ Nano-materials $\cdot$ Size effect

\section{Introduction}

An interface can markedly differ from its surrounding bulk due to processes such as aging or atomic rearrangement that can substantially affect the overall response of the body. Within a continuum mechanics setting interface behavior is often described using one of the following models:

- For non-coherent interfaces, often described by cohesive zone model (CZM), the constitutive behavior is represented by a traction-separation law. This approach is essentially based on the fact that local material damage takes place associated with a displacement jump across the interface. Thus in this approach the interface traction is a function of the displacement jump. However this model can not capture the interface mechanical response if the loading conditions do not cause any form of opening 


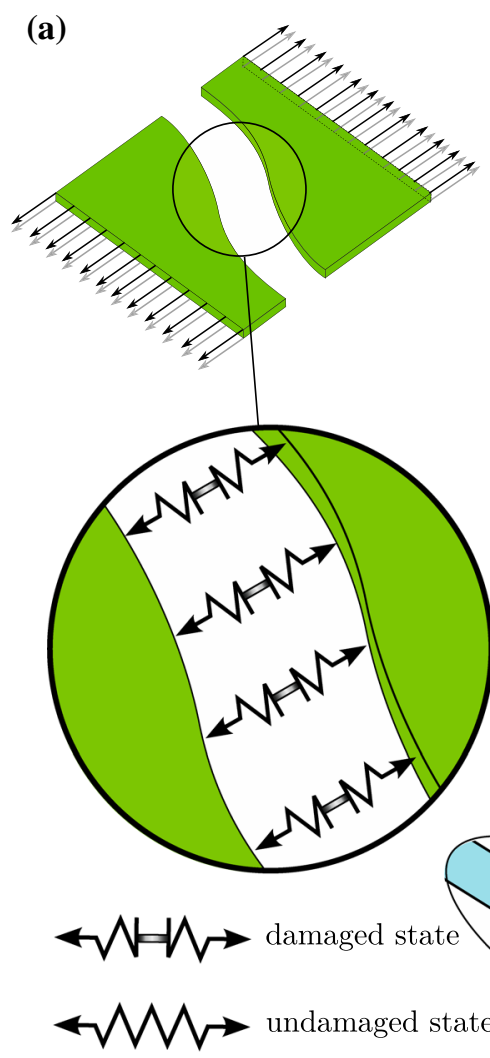

cohesive zone model (b)

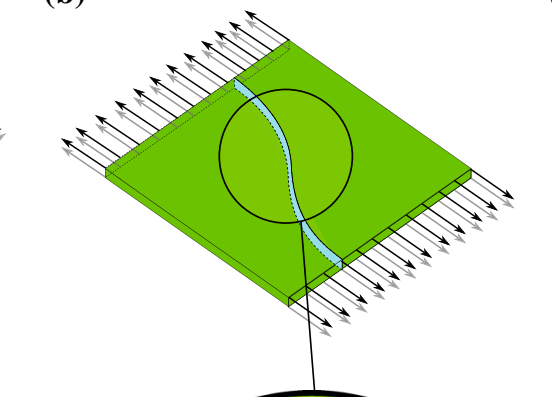

(c)

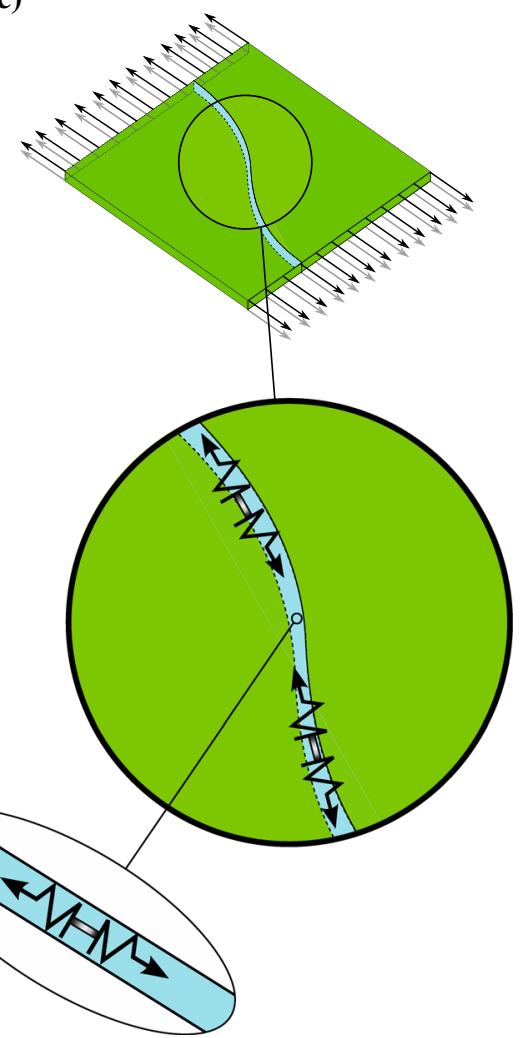

interface elasticity theory $+\mathrm{CDM}$ current contribution
Fig. 1 a Classical cohesive non-coherent interface damage model, b interface elasticity theory, $\mathbf{c}$ interface elasticity theory accounting for damage. The different color of the interface in $\mathbf{b}$

displacement. This is due to the face that classical CZM lacks any elastic resistance against tangential deformation of the interface ${ }^{1}$ and consequently can not capture the damage of the interface under such condition (see Fig. 1a). For further details on

\footnotetext{
1 The tangential deformation of the interface and shear/sliding displacement jump across the non-coherent interface (see Tvergaard 1990, for instance) are two very different phenomena. The former is measured in terms of a second-order superficial deformation gradient and the latter in terms of the displacement jump vector. The former then causes interface stress on the tangential plane of the interface resulting in the superficial second-order Piola stress tensor while the latter causes traction, a vector quantity across the interface. To induce stress on the tangential plane of the interface one needs to apply some form of deformation on the elastic interface, whereas a cohesive interface is existent if and only if there is some form of opening (normal or shear) across the interface.
}

and $\mathbf{c}$ represents the different interface material properties compared to those of the bulk. The broken and intact springs represent damaged and undamaged states of the interface respectively

cohesive damage type interface models see Barenblatt (1962), Needleman (1990, 1992, 2014), van den Bosch et al. (2006, 2007), Mosler and Scheider (2011), Aragón et al. (2013), Wu et al. (2014) and references therein.

- A coherent interface is endowed with its own elastic behavior or more precisely with its own energetic structure. Recall that the coherence condition on the interface implies the continuity of the displacement across the interface and thus the displacement jump vanishes identically. The interface elastic response is a function of the superficial interface deformation gradient. This approach, referred to as "interface elasticity theory", proves to be a very powerful tool to capture the material behavior at the nanometer scale where the interface area to the bulk volume 
ratio is significant. However, the classical interface elasticity theory suffers from the fact that the interface behavior remains elastic regardless of the strain level at the interface (see Fig. 1b). Section 1.1 briefly reviews this model.

The objective of this contribution is to extend the latter approach, i.e. interface elasticity, such that it also accounts for damage along the coherent interface (see Fig. 1c). This is motivated by the fact that the wellestablished interface elasticity theory is essentially the interface counterpart of bulk elasticity theory.

It is worth mentioning that one of the motivations behind non-local models in the bulk is mesh-objective finite element simulation of strain softening materials. Strain softening however in the local form violates Drucker's stability condition and well-posedness of the boundary value problem (Bažant and Jirásek 2002). In addition, the finite element simulations of local strain softening problems suffers from a pathological mesh dependency leading to strain localization into a zerovolume zone. In other words, converging to a meaningful solution upon mesh refinement is not achievable. Thus, non-local damage models were developed to limit localization, to guarantee the well-posedness of the problem and mesh objectivity. Furthermore, it is shown by Bažant and Xi (1991) that growth of micro-cracks is determined by the energy release from the volume encompassing the micro-crack. For further details on non-local formulation specific to plasticity and damage see Bažant and Jirásek (2002) and references therein. It is also of importance to note that the existence of a micro-crack could intensify the stress level of other neighboring micro-cracks or even function as a barrier to them. We also point out that regarding surface/interface elasticity, the connection between surface elasticity theory of Gurtin-Murdoch and nonlocal elasticity is still an open question. Therefore it is not yet clear whether or not the size effects that nonlocal and surface/interface elasticity models try to capture are the same or in any way linked. For further details see Cordero et al. (2015). Noting

- the above arguments clarifying the necessity of enriching local continuum mechanics with nonlocality,

- the fact that the local damage across the interface is not causing any problem and the non-locality of the interface damage is along the interface,
- in principle, in this contribution, we derive an interface damage model as a natural counterpart to the bulk damage model,

the damage model is non-localized and the nonlocalization is chosen to be integral-type.

The interface in this work is material indicating that the motion of the interface is bound to its surrounding bulk and thus the interface does not move independently of the bulk. The two main ingredients of the work presented here are (i) interface elasticity and (ii) damage. A brief review of these topics is now given.

\subsection{State-of-the-art review of interface elasticity}

The influence of interfaces on the overall response of materials is pronounced in small scale solids due to the large area to bulk volume ratio. The same holds for surfaces, and indeed the here considered (coherent) interfaces shall be understood as two-sided surfaces. The widely adopted surface elasticity theory of Gurtin and Murdoch (1975) and variants thereof such as interface elasticity theory (Murdoch 1976) endow the surface/interface with their own tensorial structures (stress and strain). These tensorial structures arise from an energetic structure and more generally a dissipative structure attributed to the surface/interface. For further details see for instance, Moeckel (1975), Daher and Maugin (1986), dell'Isola and Romano (1987), Cammarata (1997), Gurtin et al. (1998), Steigmann and Ogden (1999), Fried and Todres (2005), Steinmann (2008), Levitas and Javanbakht (2010), Javili and Steinmann (2010b), Cordero et al. (2015) and references therein. McBride et al. (2011) presented a non-linear continuum thermodynamics formulation accounting for diffusion and viscoelasticity effects for both bulk and energetic surfaces.

The effect of interface energetics, e.g. for inclusions, and the size-dependent elastic state of the material has been widely investigated recently for instance in Benveniste and Miloh (2001), Sharma et al. (2003), Sharma and Ganti (2004), Dingreville et al. (2005), Sharma and Wheeler (2007), Duan et al. (2005a, b), Benveniste (2006), Duan and Karihaloo (2007), Duan et al. (2009), Benveniste (2013), Huang and Sun (2007), Fischer and Svoboda (2010), Yvonnet et al. (2011a), Davydov et al. (2013) and references therein.

The finite element implementation of the lowerdimensional elasticity theory has been realized in 
Javili and Steinmann (2009, 2010a) and further extended to interface multi-physics in Javili et al. (2012, 2013b, 2014a). For a unifying review of different approaches including surface, interface and curve energies see Javili et al. (2013c).

\subsection{State-of-the-art review of continuum damage}

The term "continuum damage mechanics (CDM)" refers to any constitutive model using internal variables to characterize a material's degradation, within the continuum mechanics framework (de Souza Neto et al. 1998) first proposed by Kachanov (1958). Rabotnov (1963) provided a physical interpretation of the damage variable. Chaboche $(1981,1984)$ proposed an anisotropic damage model. Murakami and Ohno (1981) used a symmetric second-order tensor to describe the damage variable. Lemaitre (1984) proposed to relate the damage variable to the reduced Young modulus. Simo and Ju (1987) developed a strainand stress-based continuum damage model. Krajcinovic and Fonseka (1981) used a vectorial representation of the damage variable. The first finite deformation continuum damage formulation was developed by Simo and Ju (1987). Later de Souza Neto et al. (1994b) provided a framework to model the Mullins effect. de Souza Neto et al. (1994a), de Souza Neto and Perić (1996), Steinmann et al. (1994) formulated an isotropic elastoplastic damage model at large deformations.

The concept of non-locality was first introduced by Eringen (1966) and was later applied to damage (Cabot and Bažant 1987; Saanouni et al. 1989; Saouridis and Mazars 1992). Bažant (1994) provided a micro-mechanical explanation of non-local averaging. Andrade et al. $(2011,2014)$ formulated an integral-type non-local damage model at large deformations. Jirasek (1998) explored different non-local formulations based on the type of non-local variables. Boundary effects in non-local models must be avoided (Pijaudier-Cabot and Grégoire 2014) which is accomplished by normalizing the weight function or using only the local material response on the boundaries (see also, Krayani et al. 2009; Pijaudier-Cabot and Dufour 2010). For the finite element framework, Pijaudier-Cabot and Huerta (1991) derived the general form of the tangent stiffness matrix for non-local isotropic damage models (see also, Askes and Sluys 2000). The consistent derivation of the tangent stiffness matrix including non-local contributions was presented in Jirásek and Patzák (2002).
In the field of composite delamination many authors e.g. Corigliano (1993), Allix and Corigliano (1996), Allix et al. (1995), Ladevèze et al. (1998), Schellekens and Borst (1993), Chaboche et al. (1997), Bolzon and Corigliano (1997), Chen et al. (1999), Mi et al. (1998), Alfano and Crisfield (2001) employed the continuum damage mechanics approach to model the degradation of non-coherent, cohesive interface elements. Cazes et al. (2009), Ijaz et al. (2014) provided a formulation for a non-local damage-type cohesive zone model. Within a thermomechanical framework Özdemir et al. (2010), Willam et al. (2004), Fagerström and Larsson (2008), Fleischhauer et al. (2013) studied the effects of degradation of the cohesive interface on thermal properties. The coupling of an interface damage model and friction is provided in (Alfano and Sacco 2006; Chaboche et al. 1997; Lin et al. 2001; Parrinello et al. 2009; Raous 2011, among others) using cohesive zone models.

\subsection{Key objectives and contributions of this work}

This contribution follows the work of Javili and Steinmann $(2009,2010$ a) by providing an extension to include the effects of material degradation both on the interface and in the bulk using continuum damage mechanics. The focus of this work is on continuum domains including geometrically coherent interfaces possessing their own energy. The coherent interface assumes a hyperelastic response for its reversible mechanical response. The material degradation in the form of a gradual loss of stiffness occurs both on the interface and in the bulk and is modeled by introducing isotropic damage variables on all the domains. To avoid the pathological mesh dependency of local damage models, integral-type non-local averaging is implemented due to its numerical effectivity. In order to calculate the non-local weighting coefficients on the interface (in deed a two-dimensional curved manifold) the concept of minimal geodesics of differential geometry is used, which involves finding the shortest arc-length of all the curves connecting two points on the interface. The quantity to be non-localized in this work is the equivalent distortion which is a scalar measure of tensorial distortion entering the interface and bulk Helmholtz energy. A computational investigation of the influence of the inelastic (damaged) energetic interface on the overall mechanical response of the solid is performed to better understand the computational aspects of the 
model. For this purpose, we devised three cases (i. damage in the bulk only, ii. on the interface only and iii. in both the bulk and interface) and compared the results with a fully elastic case. In this manuscript the term equivalent distortion instead of the customary equivalent strain is used but in principle furnishes the same information. In summary, the key contributions of this work are as follows:

- To review the governing equations of a body possessing an energetic coherent interface in a finitedeformation setting, extend them to include continuum damage on the interface and to derive the weak form of the local balance of forces.

- To present a thermodynamically consistent formulation and derive the dissipation inequality on the interface.

- To present the calculation of minimal geodesic distance on general curved two-dimensional manifolds embedded in three-dimensional Euclidean space and particularize it to the interface geometry.

- To derive the consistent tangent stiffness matrices in the bulk and on the interface.

- To provide a finite element algorithmic setup for damage model.

- To illustrate the theory with the help of numerical examples using the finite element method.

\subsection{Organization of this manuscript}

This manuscript is organized as follows. First the notation and certain key concepts are briefly introduced. Section 2 summarizes the kinematics and the governing balance equations of non-linear continuum mechanics. A non-local continuum damage model in the bulk is briefly presented in Sect. 3. A non-local continuum damage model on the interface is derived in Sect. 4 based on the concept of effective stress and the hypothesis of strain equivalence. An interface Helmholtz energy and its arguments are introduced. Thermodynamically consistent constitutive relations are determined. A numerical framework that encompasses interface elasticity and non-local damage both in the bulk and on the interface is established in Sect. 5. The framework includes the weak formulation of the governing equations, the corresponding finite element implementation and the derivation of the consistent stiffness matrices. A series of numerical examples, based on the finite element approximation of the weak form, is presented in Sect. 6 to elucidate the theory. Section 7 concludes this work.

\subsection{Notation and definitions}

Direct notation is adopted throughout. Occasional use is made of index notation, the summation convention for repeated indices being implied. The threedimensional Euclidean space is denoted $\mathbb{E}^{3}$. The scalar product of two vectors $\boldsymbol{a}$ and $\boldsymbol{b}$ is denoted $\boldsymbol{a} \cdot \boldsymbol{b}=$ $[\boldsymbol{a}]_{i}[\boldsymbol{b}]_{i}$. The scalar product of two second-order tensors $\boldsymbol{A}$ and $\boldsymbol{B}$ is denoted $\boldsymbol{A}: \boldsymbol{B}=[\boldsymbol{A}]_{i j}[\boldsymbol{B}]_{i j}$. The composition of two second-order tensors $\boldsymbol{A}$ and $\boldsymbol{B}$, denoted $\boldsymbol{A} \cdot \boldsymbol{B}$, is a second-order tensor with components $[\boldsymbol{A} \cdot \boldsymbol{B}]_{i j}=[\boldsymbol{A}]_{i m}[\boldsymbol{B}]_{m j}$. The vector product of two vectors $\boldsymbol{a}$ and $\boldsymbol{b}$ is denoted $\boldsymbol{a} \times \boldsymbol{b}$ with $[\boldsymbol{a} \times \boldsymbol{b}]_{k}=$ $[\boldsymbol{\varepsilon}]_{i j k}[\boldsymbol{a}]_{i}[\boldsymbol{b}]_{j}$ where $\boldsymbol{\varepsilon}$ denotes the third-order permutation (Levi-Civita) tensor. The non-standard product of a fourth-order tensor $\mathbb{C}$ and a vector $\boldsymbol{b}$ is defined by $[\boldsymbol{b}: \mathbb{C}]_{i k l}=[\mathbb{C}]_{i j k l}[\boldsymbol{b}]_{j}$. The action of a secondorder tensor $\boldsymbol{A}$ on a vector $\boldsymbol{a}$ is given by $[\boldsymbol{A} \cdot \boldsymbol{a}]_{i}=$ $[\boldsymbol{A}]_{i j}[\boldsymbol{a}]_{j}$. The standard product of a fourth-order tensor $\mathbb{C}$ and a second-order tensor $\boldsymbol{A}$ is defined by $[\mathbb{C}$ : $\boldsymbol{A}]_{i j}=[\mathbb{C}]_{i j k l}[\boldsymbol{A}]_{k l}$. The dyadic product of two vectors $\boldsymbol{a}$ and $\boldsymbol{b}$ is a second-order tensor $\boldsymbol{D}=\boldsymbol{a} \otimes \boldsymbol{b}$ with $[\boldsymbol{D}]_{i j}=[\boldsymbol{a}]_{i}[\boldsymbol{b}]_{j}$. Two non-standard dyadic products of two second-order tensors $\boldsymbol{A}$ and $\boldsymbol{B}$ are the fourth-order tensors $[\boldsymbol{A} \bar{\otimes} \boldsymbol{B}]_{i j k l}=[\boldsymbol{A}]_{i k}[\boldsymbol{B}]_{j l}$ and $[\boldsymbol{A} \otimes \boldsymbol{B}]_{i j k l}=$ $[\boldsymbol{A}]_{i l}[\boldsymbol{B}]_{j k}$. The average and jump of a quantity $\{\bullet\}$ over the interface are defined by $\{\{\bullet\}\}=\frac{1}{2}\left[\{\bullet\}^{+}+\{\bullet\}^{-}\right]$ and $\llbracket\{\bullet\} \rrbracket=\{\bullet\}^{+}-\{\bullet\}^{-}$respectively. Table 1 gathers a list of notations frequently used in this manuscript.

\section{Problem definition}

This section summarizes the kinematics of non-linear continuum mechanics including material coherent interfaces and introduces the notation adopted here. Further details on the kinematics of deformable interfaces can be found in Javili et al. (2013b).

Consider a continuum body $\mathscr{B}$ that takes the material configuration $\mathcal{B}_{0} \subset \mathbb{E}^{3}$ at time $t=0$ and the spatial configuration $\mathcal{B}_{t}$ at $t>0$, as depicted in Fig. 2. The body $\mathscr{B}$ is partitioned into two disjoint subdomains, $\mathcal{B}_{0}^{+}$ and $\mathcal{B}_{0}^{-}$, by an interface $\mathcal{I}_{0}$, thus the bulk is defined by $\mathcal{B}_{0}:=\mathcal{B}_{0}^{+} \cup \mathcal{B}_{0}^{-}$with reference placements of material 
Table 1 List of important notations

\begin{tabular}{|c|c|c|c|}
\hline \multicolumn{2}{|l|}{ Bulk } & \multicolumn{2}{|c|}{ Interface } \\
\hline $\boldsymbol{F}$ & Material deformation gradient & $\bar{F}$ & Material deformation gradient \\
\hline$\varphi$ & Deformation map & $\bar{\varphi}$ & Deformation map \\
\hline$X$ & Material coordinates & $\bar{X}$ & Material coordinates \\
\hline$x$ & Spatial coordinates & $\bar{x}$ & Spatial coordinates \\
\hline$N$ & Material normal to boundary & $\bar{N}$ & Material normal to interface \\
\hline$n$ & Spatial normal to boundary & $\bar{n}$ & Spatial normal to interface \\
\hline$P$ & Piola stress & $\bar{P}$ & Piola stress \\
\hline $\boldsymbol{P}_{0}$ & Undamaged Piola stress & $\overline{\boldsymbol{P}}_{0}$ & Undamaged Piola stress \\
\hline$\Psi$ & Helmholtz energy & $\bar{\Psi}$ & Helmholtz energy \\
\hline$\Psi_{0}$ & Undamaged Helmholtz energy & $\bar{\Psi}_{0}$ & Undamaged Helmholtz energy \\
\hline$D$ & Damage parameter & $\bar{D}$ & Damage parameter \\
\hline$F_{\text {loc }}$ & Local equivalent distortion & $\bar{F}_{\mathrm{loc}}$ & Local equivalent distortion \\
\hline$F_{\text {nloc }}$ & Non-local equivalent distortion & $\bar{F}_{\text {nloc }}$ & Non-local equivalent distortion \\
\hline$F_{\max }$ & Maximum attained $F_{\text {nloc }}$ & $\bar{F}_{\max }$ & Maximum attained $\bar{F}_{\text {nloc }}$ \\
\hline
\end{tabular}

Only interface

$\begin{array}{llll}\boldsymbol{\varphi}^{ \pm} & \text {Deformation maps of } \pm \text { sides } & \boldsymbol{x}^{ \pm} & \text {Spatial coordinates of } \pm \text { sides } \\ \widetilde{\boldsymbol{n}} & \text { Spatial normal to interface boundary } & \widetilde{N} & \text { Material normal to interface boundary }\end{array}$

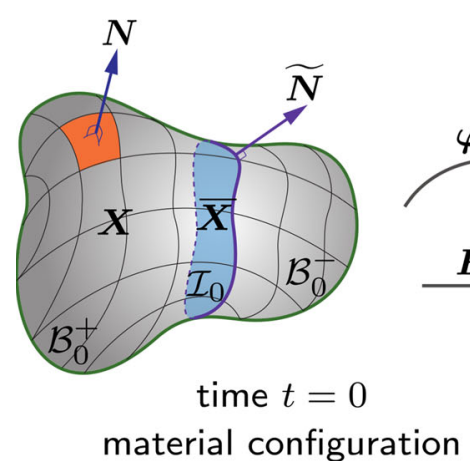

Fig. 2 The bulk domain $\mathcal{B}_{0}$, the bulk subdomains $\mathcal{B}_{0}^{ \pm}$and interface $\mathcal{I}_{0}$ and the unit normals to the surface $N$, interface $\bar{N}$ and boundary of the interface $\widetilde{N}$ all defined in the material configuration. The bulk and interface deformation maps denoted as $\varphi$ and $\bar{\varphi}$, respectively map the material configuration to the spatial configuration at time $t$. The bulk domain $\mathcal{B}_{t}$, the bulk subdo-

particles labeled $X$. The two sides of the interface $\mathcal{I}_{0}$ are denoted $\mathcal{I}_{0}^{+}:=\partial \mathcal{B}_{0}^{+} \bigcap \mathcal{I}_{0}$ and $\mathcal{I}^{-}:=\partial \mathcal{B}_{0}^{-} \bigcap \mathcal{I}_{0}$. The material particles on the interface are labeled $\bar{X}$. The outward unit normal to $\partial \mathcal{B}_{0}$ is denoted $N$. The outward
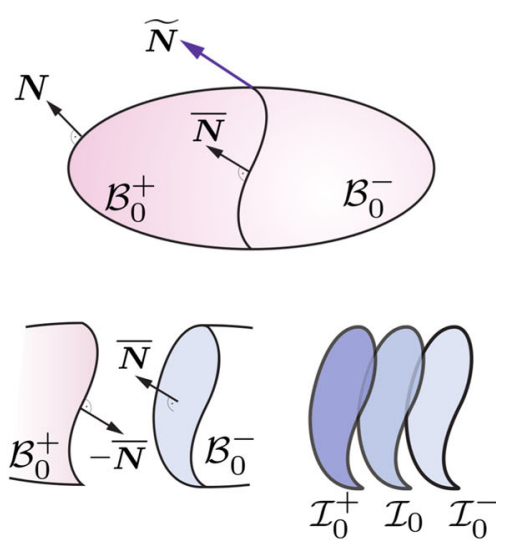

mains $\mathcal{B}_{t}^{ \pm}$and interface $\mathcal{I}_{t}$ and the unit normals to the surface $\boldsymbol{n}$, interface $\overline{\boldsymbol{n}}$ and boundary of the interface $\widetilde{\boldsymbol{n}}$ all defined in the spatial configuration. The interface unit normal is pointing from the negative side of the interface to the positive side. The bulk and rank-deficient interface deformation gradients are $\boldsymbol{F}$ and $\overline{\boldsymbol{F}}$ respectively

unit normal to the boundary of the interface $\partial \mathcal{I}_{0}$, tangent to the interface $\mathcal{I}_{0}$ is denoted $\widetilde{N}$. The unit normal to $\mathcal{I}_{0}$ is denoted $\bar{N}=\bar{N}^{-}$whose direction is conventionally taken to point from the negative side of the 
Table 2 Localized force and moment balances in the bulk and on the interface in the material configuration

\begin{tabular}{llll}
\hline Force balance & $\operatorname{Div} \boldsymbol{P}+\boldsymbol{B}^{\mathrm{p}}=\mathbf{0}$ & in $\mathcal{B}_{0}$ & $\widehat{\boldsymbol{B}}^{\mathrm{p}}=\boldsymbol{P} \cdot \boldsymbol{N}$ \\
& $\overline{\operatorname{Div}} \overline{\boldsymbol{P}}+\overline{\boldsymbol{B}}^{\mathrm{p}}+\llbracket \boldsymbol{P} \rrbracket \cdot \overline{\boldsymbol{N}}=\mathbf{0}$ & on $\mathcal{I}_{0}$ & $\widetilde{\boldsymbol{B}}^{\mathrm{p}}=\overline{\boldsymbol{P}} \cdot \widetilde{\boldsymbol{N}}$ \\
Moment balance & $\boldsymbol{P} \cdot \boldsymbol{F}^{t}=\boldsymbol{F} \cdot \boldsymbol{P}^{t}$ & in $\mathcal{B}_{0}$ & \\
& $\overline{\boldsymbol{P}} \cdot \overline{\boldsymbol{F}}^{t}=\overline{\boldsymbol{F}} \cdot \overline{\boldsymbol{P}}^{t}$ & on $\mathcal{I}_{0}$ &
\end{tabular}

The notation $\{\bullet\}^{\mathrm{p}}$ is to distinguish prescribed quantities. The notation $\{\bullet\}^{t}$ is the transposition operator. $\boldsymbol{B}^{\mathrm{p}}$ : force vector per unit volume; $\widehat{\boldsymbol{B}}^{\mathrm{p}}$ : surface traction per unit area; $\overline{\boldsymbol{B}}^{\mathrm{p}}$ : force vector per unit area; $\widetilde{\boldsymbol{B}}^{\mathrm{p}}$ : curve traction per unit length

interface to the positive side. The spatial counterparts of the various unit normals are $\boldsymbol{n}, \widetilde{\boldsymbol{n}}$ and $\overline{\boldsymbol{n}}$, respectively. The deformation maps of the bulk, and the negative and positive sides of the interface are denoted $\varphi, \varphi^{-}$ and $\varphi^{+}$, respectively. The restriction of the motion $\varphi$ to the interface is defined by $\bar{\varphi}:=\{\varphi\}$. The current placements of particles in the bulk and on the two sides of the interface are denoted $\boldsymbol{x}$ and $\boldsymbol{x}^{\mp}$ where spatial particles on the interface are designated as $\overline{\boldsymbol{x}}:=\{\boldsymbol{x}\}$. One should note that $\varphi^{+}=\varphi^{-}$and $x^{+}=x^{-}$for coherent interfaces.

The bulk and the rank-deficient interface deformation gradients are respectively defined by

$$
\begin{aligned}
& \boldsymbol{F}(\boldsymbol{X}, t):=\operatorname{Grad} \boldsymbol{\varphi}(\boldsymbol{X}, t) \quad \text { and } \\
& \overline{\boldsymbol{F}}(\overline{\boldsymbol{X}}, t):=\overline{\operatorname{Grad}} \overline{\boldsymbol{\varphi}}(\overline{\boldsymbol{X}}, t),
\end{aligned}
$$

respectively, The interface gradient and divergence operators are respectively defined by

$$
\begin{aligned}
& \overline{\operatorname{Grad}}\{\bar{\bullet}\}:=\operatorname{Grad}\{\bar{\bullet}\} \cdot \overline{\boldsymbol{I}} \quad \text { and } \\
& \overline{\operatorname{Div}}\{\bar{\bullet}\}:=\overline{\operatorname{Grad}}\{\bar{\bullet}\}: \overline{\boldsymbol{I}},
\end{aligned}
$$

with $\overline{\boldsymbol{I}}:=\boldsymbol{I}-\overline{\boldsymbol{N}} \otimes \overline{\boldsymbol{N}}$, where $\overline{\boldsymbol{I}}$ and $\boldsymbol{I}$ denote the interface and bulk unit tensors. Their spatial counterparts are denoted $\overline{\boldsymbol{i}}$ and $\boldsymbol{i}$. Finally the bulk and interface Jacobians are denoted by $J:=\operatorname{det} \boldsymbol{F}>0$ and $\bar{J}:=\overline{\operatorname{det}} \overline{\boldsymbol{F}}>0$ respectively, with $\overline{\operatorname{det}}\{\bullet\}$ denoting the area determinant (Steinmann 2008).

Equilibrium conditions in the bulk and on the interface together with associated boundary conditions are listed in Table 2 (see Javili et al. 2013b, c, for further details). A detailed derivation of balance of forces on the interface is given in section "Balance of forces on interface" of "Appendix 1". The interface Piola stress tensor $\overline{\boldsymbol{P}}$ is a superficial ${ }^{2}$ tensor field possessing the

\footnotetext{
2 The superficiality of the interface Piola stress tensor is a classical assumption of interface elasticity theory. Recently, Javili et al. (2013a) have proven that this condition is the consequence of a first-order continuum theory.
}

property $\overline{\boldsymbol{P}} \cdot \overline{\boldsymbol{N}}=\mathbf{0}$. In the absence of $\overline{\boldsymbol{B}}^{\mathrm{p}}$, the jump of traction across the interface equates with the negative divergence of the interface stress tensor. Therefore, the classical traction continuity across the interface $(\llbracket \boldsymbol{P} \rrbracket \cdot \overline{\boldsymbol{N}}=\mathbf{0})$ no longer holds. One should note that $\boldsymbol{P}$ and $\overline{\boldsymbol{P}}$ represent the general (nominal) stress states in the bulk and on the interface. This matter will be detailed in the upcoming sections.

\section{Damage model in the bulk}

In this section a brief review of a simple isotropic local damage model for the bulk is given and the non-local version of such a model is introduced. The introduced damage model is standard and the details are omitted by referring to the appropriate references.

To proceed, continuum damage is utilized resulting in a model that describes the macroscopic behavior of degrading materials containing microcracks or microvoids by introducing an internal variable denoted by $D$. The damage variable maps the nominal (damaged) stress tensor $\boldsymbol{P}$ onto the effective (undamaged) stress tensor $\boldsymbol{P}_{0}$ according to

$\boldsymbol{P}_{0}=\frac{\boldsymbol{P}}{[1-D]}$,

where $[1-D]$ is the reduction factor first introduced by Kachanov (1958). It is important to mention that the damage variable can also be understood as the ratio of the damaged surface to the total surface at a material point (Simo and Ju 1987).

To further develop the model, a damage criterion $\phi$ depending on a scalar measure $F_{\text {loc }}$ and an internal variable $F_{\max }$ controlling the evolution of damage variable $D=\mathscr{D}\left(F_{\max }\right)$ established (Simo and Ju 1987; Holzapfel 2000). Such a function typically takes the form 
$\phi\left(F_{\text {loc }}, F_{\max }\right)=F_{\text {loc }}(\boldsymbol{F})-F_{\max } \leq 0$ with

$F_{\mathrm{loc}}=\sqrt{\frac{2 Y}{E}}$ and $F_{\max }(t)=\max _{s \in[0, t]}\left\{F_{0},\left.F_{\mathrm{loc}}\right|_{s}\right\}$,

where $F_{\text {loc }}$ is the local equivalent distortion, $F_{\max }(t)$ is the maximum attained equivalent distortion in the history of deformation, $Y:=\Psi_{0}(\boldsymbol{F})$ is the thermodynamic force conjugate to the damage variable (Holzapfel 2000) and $E$ is the bulk Young's modulus. One should note that the equivalent distortion $F_{\text {loc }}$ is defined in a similar fashion to that of Jirásek and Patzák (2002). This choice for a geometrically linear one-dimensional problem results in exactly the elastic strain when assuming that the strain energy is $Y:=\frac{1}{2} E \epsilon^{2}$ with $\epsilon$ denoting the strain measure. The formulation presented in the current work, therefore, boils down to its small strain version in the limit of infinitesimal strain. Clearly, the equivalent distortion $(4)_{2}$ can be defined differently without any loss of generality.

Next, the non-local damage model based on integral averaging is presented by applying non-locality to the local equivalent distortion (Jirásek and Patzák 2002) obtaining the non-local equivalent distortion $F_{\text {nloc }}$ as follows

$$
\begin{aligned}
& F_{\text {nloc }}\left(\boldsymbol{x}_{\mathrm{r}}\right)=\int_{\mathcal{B}_{0}} \omega\left(\boldsymbol{x}_{\mathrm{r}}, \boldsymbol{x}_{\mathrm{s}}\right) F_{\text {loc }}\left(\boldsymbol{x}_{\mathrm{s}}\right) \mathrm{d} V \text { with } \\
& \omega\left(\boldsymbol{x}_{\mathrm{r}}, \boldsymbol{x}_{\mathrm{s}}\right)=\frac{\omega_{0}(r)}{\int_{\mathcal{B}_{0}} \omega_{0}(r) \mathrm{d} V},
\end{aligned}
$$

where $\omega, \omega_{0}$ and $r$ are the weight function, a nonnegative, monotonically decreasing function and a measure of distance between source points $\boldsymbol{x}_{\mathrm{s}}$ and receiver points $\boldsymbol{x}_{\mathrm{r}}$ respectively.

Finally the Kuhn-Tucker loading/unloading conditions for the bulk damage model read (Simo and Ju 1987)

$\phi \leq 0, \quad \dot{F}_{\text {max }} \geq 0, \quad \dot{F}_{\text {max }} \phi=0$,

where $\dot{F}_{\text {max }} \geq 0$ takes the role of the bulk damage consistency parameter. The bulk non-local damage relations are gathered in Table 3.

\section{Damage model on the interface}

In this section a rate-independent isotropic local damage model for large deformations on the interface is presented. Furthermore a non-local interface damage model is developed by modifying the local model in terms of integral averaging along the interface. We emphasize that an interface damage model is not available in the literature to the knowledge of the authors, therefore more details compared to the damage model in the bulk are given here.

Physically, the nucleation, growth and coalescence of microcracks are the reasons for the degradation of material properties. Considering continuum mechanics, this process (initiation of microcracks, microvoids and material degradation) can be modeled by introducing an internal variable denoted by $\bar{D}$.

To proceed a Helmholtz energy is considered for the interface containing the following arguments (Simo and Hughes 1998)

$\bar{\Psi}(\overline{\boldsymbol{F}}, \bar{D}, \bar{\kappa})=[1-\bar{D}] \bar{\Psi}_{0}(\overline{\boldsymbol{F}})+\int_{0}^{\bar{\kappa}} \overline{\mathcal{H}}\left(\bar{\kappa}^{*}\right) \mathrm{d} \bar{\kappa}^{*}$,

where $\bar{D} \in[0,1], \bar{\Psi}_{0}(\bar{F})$ is the interface effective (undamaged) Helmholtz energy, $[1-\bar{D}]$ is the interface reduction factor and $\overline{\mathcal{H}}(\bar{\kappa})$ denotes a monotonically increasing function depending on the internal variable $\bar{\kappa}$.

Remark 1 The integral term in Eq. (7) is introduced in analogy with that of Simo and Hughes (1998, section 1.3.3) and is the energy storage in the material due to the accumulation of microscopic defects. These defects cause changes in strain and stress level of the material surrounding the defects. The work done due to the presence of such defects is then added to the energy of the system. The rate of this quantity provides the driving force conjugate to the internal variable $\bar{\kappa}$ and is used to motivate a damage condition. The introduction of this term into the effective Helmholtz energy in Eq. (7) then sets the stage to derive thermodynamically consistent damage conditions and evolution laws.

Differentiating Eq. (7) with respect to time and particularizing the Clausius-Plank inequality one finds

$$
\begin{aligned}
\overline{\mathcal{D}}_{\text {int }}= & {\left[\overline{\boldsymbol{P}}-[1-\bar{D}] \frac{\partial \bar{\Psi}_{0}(\overline{\boldsymbol{F}})}{\partial \overline{\boldsymbol{F}}}\right]: \dot{\overline{\boldsymbol{F}}} } \\
& +\bar{\Psi}_{0}(\overline{\boldsymbol{F}}) \dot{\bar{D}}-\overline{\mathcal{H}}(\bar{\kappa}) \dot{\bar{\kappa}} \geq 0 .
\end{aligned}
$$

Therefore, the interface nominal Piola stress tensor $\overline{\boldsymbol{P}}$ and the reduced dissipation $\overline{\mathcal{D}}_{\text {red }}$ are expressed as 
$\overline{\boldsymbol{P}}=[1-\bar{D}] \overline{\boldsymbol{P}}_{0} \quad$ with $\quad \overline{\boldsymbol{P}}_{0}=\frac{\partial \bar{\Psi}_{0}(\overline{\boldsymbol{F}})}{\partial \overline{\boldsymbol{F}}}$,

$\overline{\mathcal{D}}_{\text {red }}=\bar{Y} \dot{\bar{D}}-\mathcal{H}(\bar{\kappa}) \dot{\bar{\kappa}} \geq 0$ with $\bar{Y}=-\frac{\partial \bar{\Psi}}{\partial \bar{D}}$,

where the quantity $\bar{Y}=\bar{\Psi}_{0}(\overline{\boldsymbol{F}})$, driving the damage evolution, is the thermodynamic force conjugate to the interface damage variable $\bar{D}$.

Next a damage condition $\bar{\Upsilon}$ is motivated as (Steinmann 1999)

$\bar{\Upsilon}(\bar{Y}, \overline{\mathcal{H}})=\bar{v}(\bar{Y})-\overline{\mathcal{H}}(\bar{\kappa}) \leq 0$,

with $\bar{v}$ being a monotonically increasing function. The Kuhn-Tucker conditions then read

$\bar{\Upsilon}(\bar{Y}, \overline{\mathcal{H}}) \leq 0, \bar{\lambda}^{*} \geq 0, \bar{\lambda}^{*} \bar{\Upsilon}(\bar{Y}, \overline{\mathcal{H}})=0$,

with $\bar{\lambda}^{*}$ being the consistency parameter. Now by defining the change of variables $\bar{F}_{\max }:=f(\bar{\kappa})$ and $\bar{F}_{\text {loc }}:=f(\bar{Y})$ and assuming $f$ to be a monotonically increasing function with the property $f(0)=0$, one obtains

$$
\begin{aligned}
& \bar{D}=\overline{\mathcal{H}}(\bar{\kappa})=\overline{\mathscr{D}}(f(\bar{\kappa}))=\overline{\mathscr{D}}\left(\bar{F}_{\max }\right) \quad \text { with } \\
& f(\bar{\kappa}(t))=\max _{s \in[0, t]}\left\{f\left(\bar{\kappa}_{0}\right),\left.f(\bar{Y})\right|_{s}\right\} \\
& \Longrightarrow \bar{F}_{\max }(t)=\max _{s \in[0, t]}\left\{\bar{F}_{0},\left.\bar{F}_{\text {loc }}\right|_{s}\right\} .
\end{aligned}
$$

Consequently, an alternative damage condition to Eq. (11) takes the form

$$
\begin{gathered}
\bar{\phi}\left(\bar{F}_{\mathrm{loc}}, \bar{F}_{\max }\right)=f\left(\bar{v}^{-1}(\bar{Y})\right)-f\left(\overline{\mathcal{H}}^{-1}(\bar{\kappa})\right) \\
=\bar{F}_{\mathrm{loc}}-\bar{F}_{\max } \leq 0 \text { with } \bar{F}_{\mathrm{loc}}:=f(\bar{Y})=\sqrt{\frac{2 \bar{Y}}{\bar{E}}},
\end{gathered}
$$

where $\bar{E}$ is the interface Young's modulus. The evolution of damage occurs when $\bar{\phi}=0$ which characterizes the damage surface.

As the final step to complete the proposed model, a non-local version of the model is presented next. A loading function $\bar{\phi}\left(\bar{F}_{\text {nloc }}, \bar{F}_{\text {max }}\right)$ and a scalar measure of the deformation gradient, the non-local equivalent distortion $\bar{F}_{\text {nloc }}$ are now introduced as follows

$$
\begin{aligned}
& \bar{\phi}\left(\bar{F}_{\text {nloc }}, \bar{F}_{\text {max }}\right)=\bar{F}_{\text {nloc }}-\bar{F}_{\text {max }} \leq 0 \quad \text { and } \\
& \bar{F}_{\text {nloc }}\left(\overline{\boldsymbol{x}}_{\mathrm{r}}\right)=\int_{\mathcal{I}_{0}} \bar{\omega}\left(\overline{\boldsymbol{x}}_{\mathrm{r}}, \overline{\boldsymbol{x}}_{\mathrm{s}}\right) \bar{F}_{\text {loc }}\left(\boldsymbol{x}_{s}\right) \mathrm{d} A,
\end{aligned}
$$

where $\bar{\omega}\left(\overline{\boldsymbol{x}}_{\mathrm{r}}, \overline{\boldsymbol{x}}_{\mathrm{s}}\right)$, a given non-local weight function depending on the geodesic distance $\bar{r}=\left\|\overline{\boldsymbol{x}}_{\mathrm{r}}-\overline{\boldsymbol{x}}_{\mathrm{s}}\right\|_{\mathcal{I}}$ between the source point $\overline{\boldsymbol{x}}_{\mathrm{s}}$ and the receiver point $\overline{\boldsymbol{x}}_{\mathrm{r}}$ is defined by

$$
\begin{aligned}
& \bar{\omega}\left(\overline{\boldsymbol{x}}_{\mathrm{r}}, \overline{\boldsymbol{x}}_{\mathrm{s}}\right)=\frac{\bar{\omega}_{0}(\bar{r})}{\int_{\mathcal{I}_{0}} \bar{\omega}_{0}(\bar{r}) \mathrm{d} A} \\
& \bar{\omega}_{0}(\bar{r})=\left\{\begin{array}{lll}
{\left[1-\bar{r}^{2} / \bar{R}^{2}\right]^{2}} & \text { if } \quad|\bar{r}| \leq \bar{R}, \\
0 & \text { if } \quad|\bar{r}| \geq \bar{R} .
\end{array}\right.
\end{aligned}
$$

with

Remark 2 The extension of the non-local damage from the three dimensional setting to lower dimensions is only straightforward when the lower-dimensional manifold is not curved. Nevertheless, the non-local damage on a two-dimensional (curved) manifold embedded in a three-dimensional Euclidean space involves the concept of minimal geodesics from differential geometry which is not common in the classical finite element method (see Fig. 3) and requires a non-standard treatment briefly addressed in section "Appendix 2". For simple cases of curved manifolds there exist closed form solutions to finding the minimal geodesics. (a)

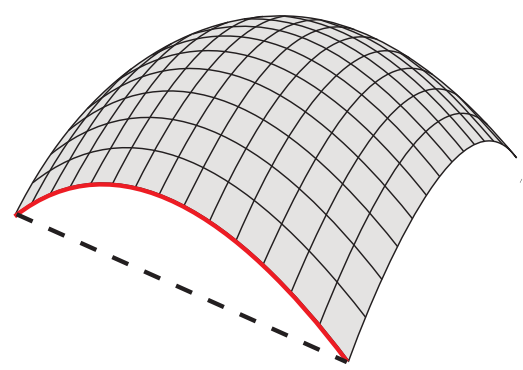

(b)

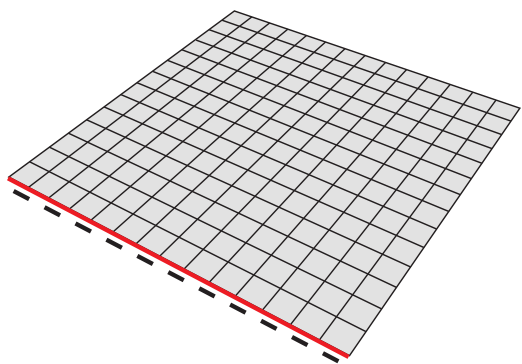

(c)

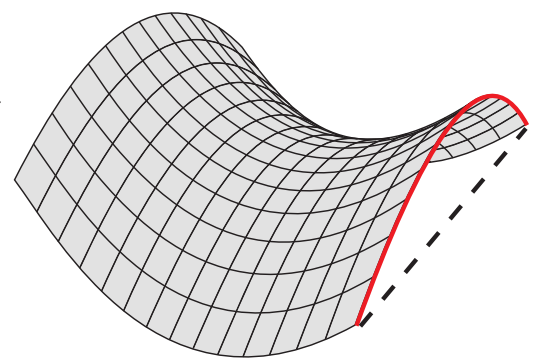

Fig. 3 The difference between geodesic (red solid curves) and straight-line Euclidean distance (black dashed lines) on curved (a), (c) and flat surfaces $(\mathbf{b})$ 
Fig. 4 Stress versus equivalent distortion diagram with exponential softening on the interface (a) and in the bulk (b). The parameters $\bar{F}_{0}$ and $\bar{F}_{\mathrm{f}}$ are the interface critical equivalent distortion and ductility response. Their bulk counterparts are denoted by $F_{0}$ and $F_{\mathrm{f}}$ respectively (a)

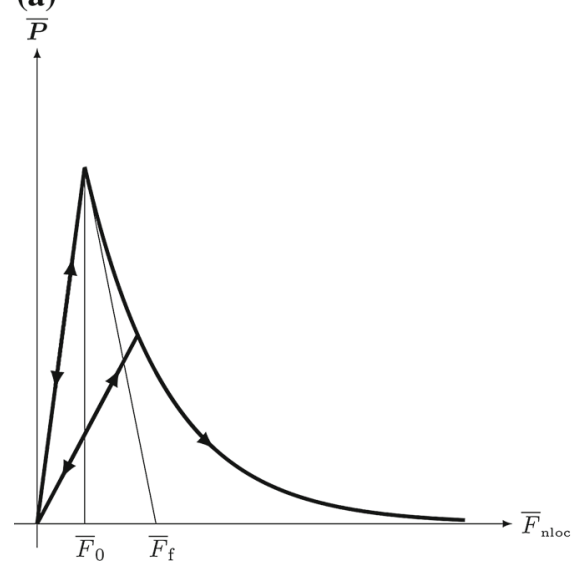

(b)

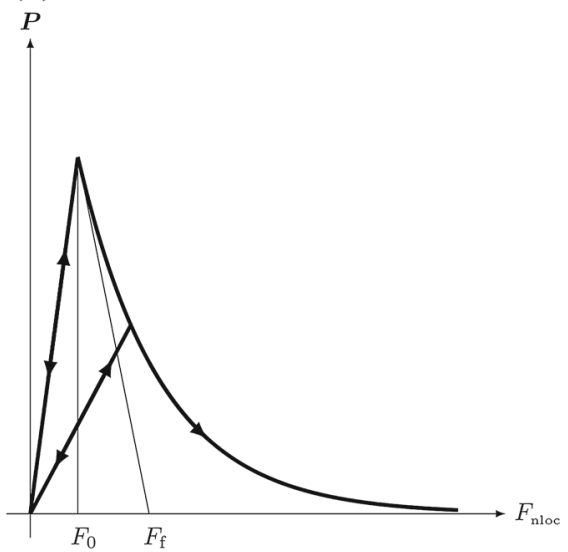

The interface interaction radius is denoted by $\bar{R}$. Note that in Eq. $(15)_{2}$, integral extends on the lowerdimensional manifold $\mathcal{I}_{0}$. The maximum attained value of $\bar{F}_{\text {nloc }}$ in the deformation history is found using Eq. (14). ${ }^{3}$ In Eq. (16) $)_{1}, \bar{\omega}$ is scaled to account for the boundary effects, where a non-negative and monotonically decreasing (for $\bar{r} \geq 0$ ) piecewise polynomial bell-shaped function $\bar{\omega}_{0}$ is introduced.

Finally, a smooth function to relate the damage variable $\bar{D}=\overline{\mathscr{D}}\left(\bar{F}_{\text {max }}\right)$ to the history variable $\bar{F}_{\text {max }}$ with an exponential softening law is chosen as follows

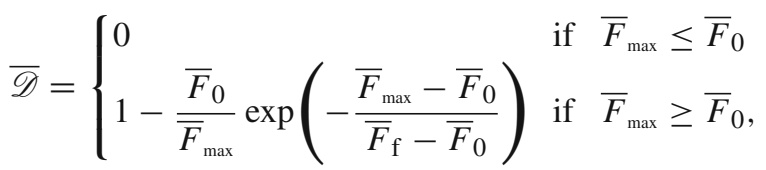

where $\bar{F}_{\mathrm{f}}$ affects the ductility of the response (see Fig. 4). All the equations required by the non-local damage model both in the bulk and on the interface are listed in Table 3.

\section{Computational framework}

The purpose of this section is to establish a numerical framework that encompasses elasticity combined with

\footnotetext{
${ }_{3}$ Note that the same notation $\bar{F}_{\max }$ is used for both the local and non-local versions. Nevertheless, they are clearly distinguished by their definition. The implementation of this contribution focuses only on the non-local version. Clearly, the nonlocal theory boils down to the local theory in the limit case of $\bar{\omega}$ being the Dirac delta distribution. This can be achieved by setting $\bar{R}=0$. The same discussion holds for the bulk as well.
}

non-local damage both in the bulk and on the interface. Deriving the weak form and temporal and spatial (finite element) discretizations will be presented next.

\subsection{Weak form}

To derive the mechanical weak form, the localized force balance equations in the bulk and on the interface given in Table 2 are tested (from the left) with vector valued test functions $\delta \varphi \in \mathscr{H}^{1}\left(\mathcal{B}_{0}\right)$ and $\delta \bar{\varphi} \in \mathscr{H}^{1}\left(\mathcal{I}_{0}\right)$, respectively. The result is then integrated over the corresponding domains in the material configuration. Using the bulk and interface divergence theorems (see "Extended divergence theorem" section of "Appendix 1") and the orthogonality properties of the interface Piola stress measures, the weak form of the balance of linear momentum is (Javili et al. 2012)

$$
\begin{aligned}
& \int_{\mathcal{B}_{0}} \boldsymbol{P}: \operatorname{Grad} \delta \boldsymbol{\varphi} \mathrm{d} V+\int_{\mathcal{I}_{0}} \overline{\boldsymbol{P}}: \overline{\operatorname{Grad}} \delta \overline{\boldsymbol{\varphi}} \mathrm{d} A \\
& -\int_{\mathcal{B}_{0}} \delta \boldsymbol{\varphi} \cdot \boldsymbol{B}^{\mathrm{p}} \mathrm{d} V-\int_{\mathcal{I}_{0}} \delta \overline{\boldsymbol{\varphi}} \cdot \overline{\boldsymbol{B}}^{\mathrm{p}} \mathrm{d} A \\
& -\int_{\partial \mathcal{B}_{0}^{\mathrm{N}}} \delta \boldsymbol{\varphi} \cdot \widehat{\boldsymbol{B}}_{\mathrm{N}}^{\mathrm{p}} \mathrm{d} A-\int_{\partial \mathcal{I}_{0}^{\mathrm{N}}} \delta \overline{\boldsymbol{\varphi}} \cdot \widetilde{\boldsymbol{B}}_{\mathrm{N}}^{\mathrm{p}} \mathrm{d} L=0, \\
& \forall \delta \boldsymbol{\varphi} \in \mathscr{H}^{1}\left(\mathcal{B}_{0}\right), \forall \delta \overline{\boldsymbol{\varphi}} \in \mathscr{H}^{1}\left(\mathcal{I}_{0}\right) \text { with } \delta \overline{\boldsymbol{\varphi}}=\left.\{\delta \boldsymbol{\varphi}\}\right|_{\mathcal{I}_{0}} .
\end{aligned}
$$

A detailed derivation of the mechanical weak form is presented in "Weak form of the balance of forces" section "Appendix 1".

Remark 3 The objectivity of the Helmholtz energy guarantees the symmetry of Cauchy stress and thus the balance of angular momentum. Therefore the weak 
Table 3 Non-local damage model relations for the bulk and the interface

\begin{tabular}{|c|c|}
\hline Bulk & Interface \\
\hline$Y=\Psi_{0}(\boldsymbol{F})=-\frac{\partial \Psi}{\partial D} \geq 0$ & $\bar{Y}=\bar{\Psi}_{0}(\overline{\boldsymbol{F}})=-\frac{\partial \bar{\Psi}}{\partial \bar{D}} \geq 0$ \\
\hline$F_{\mathrm{loc}}=\sqrt{\frac{2 \mathrm{Y}}{E}}, r=\left\|x_{\mathrm{r}}-x_{\mathrm{s}}\right\|$ & $\bar{F}_{\mathrm{loc}}=\sqrt{\frac{2 \bar{Y}}{\bar{E}}}, \bar{r}=\left\|\overline{\boldsymbol{x}}_{\mathrm{r}}-\overline{\boldsymbol{x}}_{\mathrm{s}}\right\|_{\mathcal{I}}$ \\
\hline$\omega_{0}(r)=\left\{\begin{array}{lll}{\left[1-\frac{r^{2}}{R^{2}}\right]^{2}} & \text { if } & |r| \leq R \\
0 & \text { if } \quad|r| \geq R\end{array}\right.$ & $\bar{\omega}_{0}(\bar{r})= \begin{cases}{\left[1-\frac{\bar{r}^{2}}{\bar{R}^{2}}\right]^{2}} & \text { if } \quad|\bar{r}| \leq \bar{R} \\
0 & \text { if } \quad|\bar{r}| \geq \bar{R}\end{cases}$ \\
\hline$\omega\left(\boldsymbol{x}_{\mathrm{r}}, \boldsymbol{x}_{\mathrm{s}}\right)=\frac{\omega_{0}(r)}{\int_{\mathcal{B}_{0}} \omega_{0}(r) \mathrm{d} V}$ & $\bar{\omega}\left(\overline{\boldsymbol{x}}_{\mathrm{r}}, \overline{\boldsymbol{x}}_{\mathrm{s}}\right)=\frac{\bar{\omega}_{0}(\bar{r})}{\int_{\mathcal{I}_{0}} \bar{\omega}_{0}(\bar{r}) \mathrm{d} A}$ \\
\hline$F_{\mathrm{nloc}}\left(\boldsymbol{x}_{\mathrm{r}}\right)=\int_{\mathcal{B}_{0}} \omega\left(\boldsymbol{x}_{\mathrm{r}}, \boldsymbol{x}_{\mathrm{s}}\right) F_{\mathrm{loc}}\left(\boldsymbol{x}_{s}\right) \mathrm{d} V$ & $\bar{F}_{\text {nloc }}\left(\overline{\boldsymbol{x}}_{\mathrm{r}}\right)=\int_{\mathcal{I}_{0}} \bar{\omega}\left(\overline{\boldsymbol{x}}_{\mathrm{r}}, \overline{\boldsymbol{x}}_{\mathrm{s}}\right) \bar{F}_{\mathrm{loc}}\left(\overline{\boldsymbol{x}}_{s}\right) \mathrm{d} A$ \\
\hline$D= \begin{cases}0 & \text { if } F_{\max } \leq F_{0} \\
1-\frac{F_{0}}{F_{\max }} \exp \left(-\frac{F_{\max }-F_{0}}{F_{\mathrm{f}}-F_{0}}\right) & \text { if } F_{\max } \geq F_{0}\end{cases}$ & $\bar{D}= \begin{cases}0 & \text { if } \bar{F}_{\text {max }} \leq \bar{F}_{0} \\
1-\frac{\bar{F}_{0}}{\bar{F}_{\text {max }}} \exp \left(-\frac{\bar{F}_{\text {max }}-\bar{F}_{0}}{\bar{F}_{\mathrm{f}}-\bar{F}_{0}}\right) & \text { if } \bar{F}_{\text {max }} \geq \bar{F}_{0}\end{cases}$ \\
\hline$F_{\max }(t)=\max _{s \in[0, t]}\left\{F_{0},\left.F_{\text {nloc }}\right|_{s}\right\}$ & $\bar{F}_{\max }(t)=\max _{s \in[0, t]}\left\{\bar{F}_{0},\left.\bar{F}_{\text {nloc }}\right|_{s}\right\}$ \\
\hline$\phi\left(F_{\text {nloc }}, F_{\max }\right)=F_{\text {nloc }}-F_{\max } \leq 0$ & $\bar{\phi}\left(\bar{F}_{\text {nloc }}, \bar{F}_{\max }\right)=\bar{F}_{\text {nloc }}-\bar{F}_{\max } \leq 0$ \\
\hline
\end{tabular}

$R$ : bulk interaction radius; $\bar{R}$ : interface interaction radius; $F_{0}$ : bulk critical distortion; $\bar{F}_{0}$ : interface critical distortion; $F_{\mathrm{f}}$ : bulk ductility response; $\bar{F}_{\mathrm{f}}$ : interface ductility response; $E$ : bulk Young's modulus; $\bar{E}$ : interface Young's modulus

form is only derived from the balance of linear momentum.

\subsection{Finite element implementation}

In order to apply the finite element method to the present problem, the weak form Eq. (18) is discretized. The discretization is carried out first in time using the finite difference scheme and subsequently in space by means of the finite element method. The finite element procedure for the bulk is standard and on the interface it mimics the methodology detailed in Javili et al. (2014b).

The fully-discrete coupled non-linear system of governing equations can be stated as follows

${ }^{\text {tot }} \mathbf{R}(\mathbf{d}) \stackrel{!}{=} \mathbf{0} \quad$ with $\quad{ }^{\text {tot }} \mathbf{R}=\mathbf{R}+\overline{\mathbf{R}}$,

where $\mathbf{d}$ is the unknown global vector of spatial coordinates and total residual vector ${ }^{\text {tot }} \mathbf{R}$ can be decomposed into the contributions from the bulk and interface $\mathbf{R}$ and $\overline{\mathbf{R}}$, respectively. To solve (19) 1 , a Newton-Raphson scheme is utilized. The consistent linearization then yields

$$
{ }^{\mathrm{tot}} \mathbf{R}(\mathbf{d})+\left.\frac{\partial^{\mathrm{tot}} \mathbf{R}}{\partial \mathbf{d}}\right|_{k} \Delta \mathbf{d}_{k} \stackrel{!}{=} \mathbf{0} \quad \text { and } \quad \mathbf{d}_{k+1}=\mathbf{d}_{k}+\Delta \mathbf{d}_{k},
$$

where $k$ is the iteration number. The corresponding total (algorithmic) tangent stiffness matrix is defined by

${ }^{\text {tot }} \mathbf{K}:=\frac{{ }^{\text {tot }} \mathbf{R}}{\partial \mathbf{d}} \quad$ with $\quad{ }^{\text {tot }} \mathbf{K}=\mathbf{K}+\overline{\mathbf{K}}$,

which can be decomposed into contributions from the bulk $\mathbf{K}$ and interface $\overline{\mathbf{K}}$.

\subsubsection{Consistent (algorithmic) stiffness matrix}

For a local damage model implementation, the consistent stiffness sub-matrices can be defined by

$$
\begin{aligned}
& \mathbf{K}^{I J}=\frac{\partial \mathbf{R}^{I}}{\partial \varphi^{J}}=\int_{\mathcal{B}_{0}} \operatorname{Grad} N^{I} \cdot \mathbb{A} \cdot \operatorname{Grad} N^{J} \mathrm{~d} V, \\
& \overline{\mathbf{K}}^{I J}=\frac{\partial \overline{\mathbf{R}}^{I}}{\partial \overline{\boldsymbol{\varphi}}^{J}}=\int_{\mathcal{I}_{0}} \overline{\operatorname{Grad}} \bar{N}^{I} \cdot \overline{\mathbb{A}} \cdot \overline{\operatorname{Grad}} \bar{N}^{J} \mathrm{~d} A,
\end{aligned}
$$


where $\mathbb{A}=\partial \boldsymbol{P} / \partial \boldsymbol{F}, \overline{\mathbb{A}}=\partial \overline{\boldsymbol{P}} / \partial \overline{\boldsymbol{F}}$, and

$$
\begin{aligned}
\mathbf{R}^{I} & =\int_{\mathcal{B}_{0}} \boldsymbol{P} \cdot \operatorname{Grad} N^{I} \mathrm{~d} V-\int_{\mathcal{B}_{0}} N^{I} \boldsymbol{B}^{\mathrm{p}} \mathrm{d} V \text { and } \\
\overline{\mathbf{R}}^{I} & =\int_{\mathcal{I}_{0}} \overline{\boldsymbol{P}} \cdot \overline{\operatorname{Grad}} \bar{N}^{I} \mathrm{~d} A .
\end{aligned}
$$

For a non-local damage model however, the consistent derivation of the bulk and the interface stiffness matrix requires special treatments which is presented next. The nodal interface ${ }^{4}$ tangent stiffness matrix $\overline{\mathbf{K}}^{I J}$ is obtained by taking the derivative $\partial \overline{\mathbf{R}}^{I} / \partial \bar{\varphi}^{J}$. Therefore it is necessary to evaluate first $\overline{\mathbb{A}}$ as follows

$\overline{\mathbb{A}}=\frac{\partial \overline{\boldsymbol{P}}}{\partial \overline{\boldsymbol{F}}}=[1-\bar{D}] \overline{\mathbb{A}}_{0}-\overline{\boldsymbol{P}}_{0} \otimes \frac{\partial \overline{\mathscr{D}}}{\partial \overline{\boldsymbol{F}}}$.

The first term on the right hand side of Eq. (26) results in a local stiffness matrix also known as secant stiffness matrix $\overline{\mathbf{K}}_{\text {sect }}^{I J}$ and is defined by

$\overline{\mathbf{K}}_{\text {sect }}^{I J}=\int_{\mathcal{I}_{0}} \overline{\operatorname{Grad}} \bar{N}^{I}\left(\overline{\boldsymbol{x}}_{\mathrm{r}}\right) \cdot \overline{\mathbb{A}}_{\text {sect }}\left(\overline{\boldsymbol{x}}_{\mathrm{r}}\right) \cdot \overline{\operatorname{Grad}} \bar{N}^{J}\left(\overline{\boldsymbol{x}}_{\mathrm{r}}\right) \mathrm{d} A_{\mathrm{r}}$,

where $\overline{\mathbb{A}}_{\text {sect }}=[1-\bar{D}] \overline{\mathbb{A}}_{0}$ and $\mathrm{d} A_{\mathrm{r}}=\mathrm{d} A\left(\overline{\boldsymbol{x}}_{\mathrm{r}}\right)$.

To find the non-local contributions on the right hand side of Eq. (26) to the global stiffness matrix, one needs to obtain the derivative of the damage variable with respect to the deformation gradient $\partial \overline{\mathscr{D}} / \partial \overline{\boldsymbol{F}}$ as follows

$\frac{\partial \overline{\mathscr{D}}}{\partial \overline{\boldsymbol{F}}}=\frac{\partial \overline{\mathscr{D}}}{\partial \bar{F}_{\text {nloc }}} \frac{\partial \bar{F}_{\text {nloc }}}{\partial \overline{\boldsymbol{F}}}=\overline{\mathscr{D}}^{\prime}\left(\bar{F}_{\text {nloc }}\right) \frac{\partial \bar{F}_{\text {nloc }}}{\partial \overline{\boldsymbol{F}}}$,

where $\overline{\mathscr{D}}^{\prime}$ is the derivative of $\overline{\mathscr{D}}$ with respect to its argument and $\overline{\mathscr{D}}^{\prime}=0$ in the case of unloading. To find $\partial \bar{F}_{\text {nloc }} / \partial \overline{\boldsymbol{F}}$, Eq. $(15)_{2}$ is differentiated with respect to the deformation gradient leading to

$$
\begin{gathered}
\frac{\partial \bar{F}_{\text {nloc }}}{\partial \overline{\boldsymbol{F}}}\left(\overline{\boldsymbol{x}}_{\mathrm{r}}\right)=\int_{\mathcal{I}_{0}} \bar{\omega}\left(\overline{\boldsymbol{x}}_{\mathrm{r}}, \overline{\boldsymbol{x}}_{\mathrm{s}}\right) \frac{\partial \bar{F}_{\mathrm{loc}}\left(\overline{\boldsymbol{x}}_{\mathrm{s}}\right)}{\partial \overline{\boldsymbol{F}}\left(\overline{\boldsymbol{x}}_{\mathrm{s}}\right)} \mathrm{d} A_{\mathrm{s}} \text { with } \\
\frac{\partial \bar{F}_{\text {loc }}}{\partial \overline{\boldsymbol{F}}}=\frac{\partial}{\partial \overline{\boldsymbol{F}}} \sqrt{2 \overline{\mathrm{Y}} / \bar{E}}=\frac{1}{\bar{E} \overline{\boldsymbol{F}}_{\mathrm{loc}}} \overline{\boldsymbol{P}}_{0},
\end{gathered}
$$

\footnotetext{
4 The derivation is only carried out for the interface tangent stiffness matrix. Analogous derivations for the bulk are standard and are omitted for the sake of conciseness.
}

where $\mathrm{d} A_{\mathrm{s}}=\mathrm{d} A\left(\overline{\boldsymbol{x}}_{\mathrm{s}}\right)$. Having $\partial \overline{\mathscr{D}} / \partial \overline{\boldsymbol{F}}$, the non-local part of interface stiffness matrix reads

$$
\begin{aligned}
\overline{\mathbf{K}}_{\mathrm{nl}}^{I J} & =\int_{\mathcal{I}_{0}} \overline{\operatorname{Grad}} \bar{N}^{I}\left(\overline{\boldsymbol{x}}_{\mathrm{r}}\right)-\overline{\mathscr{D}}^{\prime}\left(\overline{\boldsymbol{x}}_{\mathrm{r}}\right) \overline{\boldsymbol{P}}_{0}\left(\overline{\boldsymbol{x}}_{\mathrm{r}}\right) \\
\otimes & {\left[\int_{\mathcal{I}_{0}} \bar{\omega}\left(\overline{\boldsymbol{x}}_{\mathrm{r}}, \overline{\boldsymbol{x}}_{\mathrm{s}}\right) \frac{\partial \overline{\boldsymbol{F}}_{\mathrm{loc}}\left(\overline{\boldsymbol{x}}_{\mathrm{s}}\right)}{\partial \overline{\boldsymbol{F}}\left(\overline{\boldsymbol{x}}_{\mathrm{s}}\right)} \cdot \overline{\operatorname{Grad}} \bar{N}^{J}\left(\overline{\boldsymbol{x}}_{\mathrm{s}}\right) \mathrm{d} A_{\mathrm{s}}\right] \mathrm{d} A_{\mathrm{r}} . }
\end{aligned}
$$

Finally the interface nodal consistent stiffness matrix takes the form

$$
\begin{aligned}
& \overline{\mathbf{K}}^{I J}=\int_{\mathcal{I}_{0}} \overline{\operatorname{Grad}} \bar{N}^{I}\left(\overline{\boldsymbol{x}}_{\mathrm{r}}\right) \cdot \overline{\mathbb{A}}_{\mathrm{sect}}\left(\overline{\boldsymbol{x}}_{\mathrm{r}}\right) \cdot \overline{\operatorname{Grad}} \bar{N}^{J}\left(\overline{\boldsymbol{x}}_{\mathrm{r}}\right) \mathrm{d} A_{\mathrm{r}} \\
&-\int_{\mathcal{I}_{0}} \overline{\operatorname{Grad}} \bar{N}^{I}\left(\overline{\boldsymbol{x}}_{\mathrm{r}}\right) \overline{\mathscr{D}^{\prime}}\left(\overline{\boldsymbol{x}}_{\mathrm{r}}\right) \overline{\boldsymbol{P}}_{0}\left(\overline{\boldsymbol{x}}_{\mathrm{r}}\right) \\
& \otimes {\left[\int_{\mathcal{I}_{0}} \bar{\omega}\left(\overline{\boldsymbol{x}}_{\mathrm{r}}, \overline{\boldsymbol{x}}_{\mathrm{s}}\right) \frac{\partial \overline{\boldsymbol{F}}_{\mathrm{loc}}\left(\overline{\boldsymbol{x}}_{\mathrm{s}}\right)}{\partial \overline{\boldsymbol{F}}\left(\overline{\boldsymbol{x}}_{\mathrm{s}}\right)} \cdot \overline{\operatorname{Grad}} \bar{N}^{J}\left(\overline{\boldsymbol{x}}_{\mathrm{s}}\right) \mathrm{d} A_{\mathrm{s}}\right] \mathrm{d} A_{\mathrm{r}} . }
\end{aligned}
$$

Similarly the bulk nodal consistent stiffness matrix is computed as

$$
\begin{aligned}
& \mathbf{K}^{I J}=\int_{\mathcal{B}_{0}} \operatorname{Grad} N^{I}\left(\boldsymbol{x}_{\mathrm{r}}\right)^{-} \mathbb{A}_{\mathrm{sect}}\left(\boldsymbol{x}_{\mathrm{r}}\right) \cdot \operatorname{Grad} N^{J}\left(\boldsymbol{x}_{\mathrm{r}}\right) \mathrm{d} V_{\mathrm{r}} \\
& \quad-\int_{\mathcal{B}_{0}} \operatorname{Grad} N^{I}\left(\boldsymbol{x}_{\mathrm{r}}\right): \mathscr{D}^{\prime}\left(\boldsymbol{x}_{\mathrm{r}}\right) \boldsymbol{P}_{0}\left(\boldsymbol{x}_{\mathrm{r}}\right) \\
& \otimes {\left[\int_{\mathcal{B}_{0}} \omega\left(\boldsymbol{x}_{\mathrm{r}}, \boldsymbol{x}_{\mathrm{s}}\right) \frac{\partial F_{\mathrm{loc}}\left(\boldsymbol{x}_{\mathrm{s}}\right)}{\partial \boldsymbol{F}\left(\boldsymbol{x}_{\mathrm{s}}\right)} \cdot \operatorname{Grad} N^{J}\left(\boldsymbol{x}_{\mathrm{s}}\right) \mathrm{d} V_{\mathrm{s}}\right] \mathrm{d} V_{\mathrm{r}}, }
\end{aligned}
$$

where $\mathbb{A}_{\text {sect }}=[1-D] \mathbb{A}_{0}, \mathrm{~d} V_{\mathrm{r}}=\mathrm{d} V\left(\boldsymbol{x}_{\mathrm{r}}\right), \mathrm{d} V_{\mathrm{s}}=$ $\mathrm{d} V\left(\boldsymbol{x}_{\mathrm{s}}\right)$ and $\mathscr{D}^{\prime}=\partial \mathscr{D} / \partial F_{\text {nloc }}$. It is important to notice the double integrals in the second terms of Eqs. (31) and (32). The reason for the presence of these terms is the non-locality of the equivalent distortion where indices $I$ and $J$ (in the non-local term) do not only belong to the nodes of the same element. Instead now index $J$ can also belong to the nodes of all the elements that fall into the influence zone of node $I$ (see Fig. 5). This introduces more nonzero elements in the stiffness matrix, however, a quadratic convergence is guaranteed due to its consistent derivation.

\subsubsection{Numerical quadrature}

Due to the nonstandard form of the stiffness integrals, their Gauss-point representations are presented as follows 


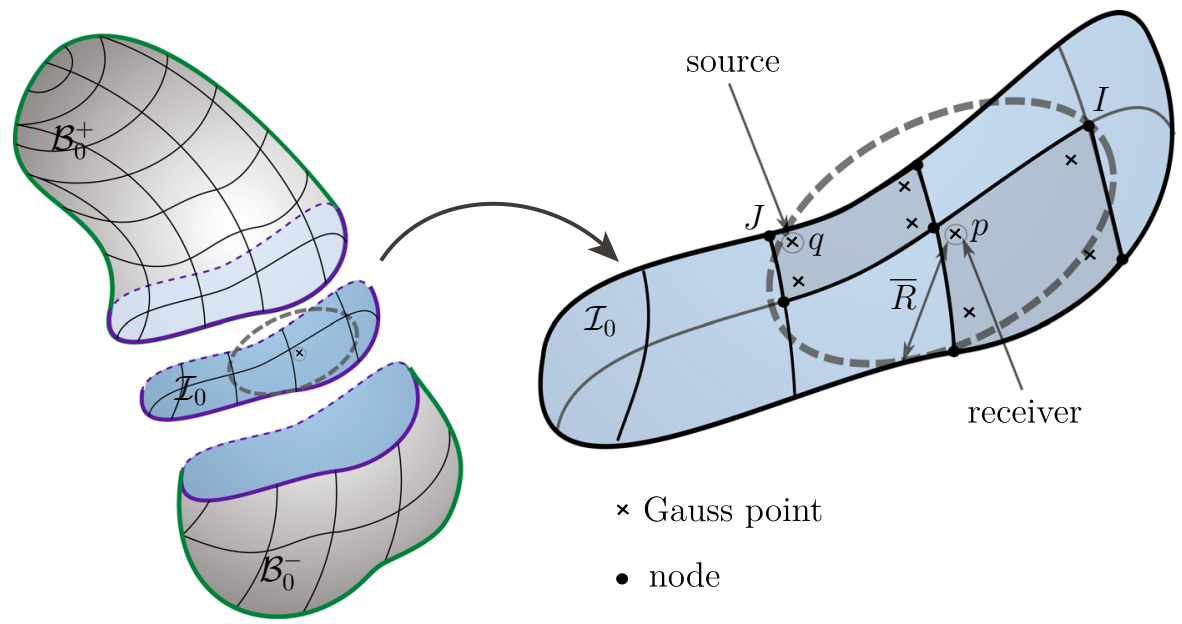

Fig. 5 Non-local interactions between a pair of the interface Gauss points $p$ and $q$. The Gauss point $p$ is the receiver of the contributions of all the source points $q$ located inside a circle centered at the node $p$ with the radius $\bar{R}$. The nodes $I$ and $J$ belong to two different elements in the stiffness assembly procedure as opposed to the standard one where these nodes belong to

$$
\begin{aligned}
& \overline{\mathbf{K}}^{I J}=\sum_{p=1}^{\bar{n}_{\text {egp }}}\left[\left[\bar{w}^{p} \overline{\mathcal{J}}\left(\overline{\boldsymbol{x}}_{\mathrm{r}}^{p}\right) \overline{\operatorname{Grad}} \bar{N}^{I}\left(\overline{\boldsymbol{x}}_{\mathrm{r}}^{p}\right)\right.\right. \\
& \left.\cdot[1-\bar{D}] \overline{\mathbb{A}}_{0}\left(\overline{\boldsymbol{x}}_{\mathrm{r}}^{p}\right) \cdot \overline{\operatorname{Grad}} \bar{N}^{J}\left(\overline{\boldsymbol{x}}_{\mathrm{r}}^{p}\right)\right] \\
& -\sum_{p=1}^{\bar{n}_{\text {egp }}} \sum_{q=1}^{\bar{n}_{\text {egp }}}\left[\bar{w}^{p} \overline{\mathcal{J}}\left(\overline{\boldsymbol{x}}_{\mathrm{r}}^{p}\right) \overline{\operatorname{Grad}} \bar{N}^{I}\left(\overline{\boldsymbol{x}}_{\mathrm{r}}^{p}\right) \cdot \overline{\mathscr{D}}^{\prime}\left(\overline{\boldsymbol{x}}_{\mathrm{r}}^{p}\right) \overline{\boldsymbol{P}}_{0}\left(\overline{\boldsymbol{x}}_{\mathrm{r}}^{p}\right)\right. \\
& \left.\left.\otimes \bar{\omega}\left(\overline{\boldsymbol{x}}_{\mathrm{r}}^{p}, \overline{\boldsymbol{x}}_{\mathrm{s}}^{q}\right) \frac{\partial \bar{F}_{\mathrm{loc}}\left(\overline{\boldsymbol{x}}_{\mathrm{s}}^{q}\right)}{\partial \overline{\boldsymbol{F}}\left(\overline{\boldsymbol{x}}_{\mathrm{s}}^{q}\right)} \cdot \overline{\operatorname{Grad}} \bar{N}^{J}\left(\overline{\boldsymbol{x}}_{\mathrm{S}}^{q}\right)\right]\right], \\
& \mathbf{K}^{I J}=\sum_{p=1}^{n_{\text {egp }}}\left[\left[w^{p} \mathcal{J}\left(\boldsymbol{x}_{\mathrm{r}}^{p}\right) \overline{\operatorname{Grad}} N^{I}\left(\boldsymbol{x}_{\mathrm{r}}^{p}\right)\right.\right. \\
& \left.\cdot[1-D] \mathbb{A}_{0}\left(\boldsymbol{x}_{\mathrm{r}}^{p}\right) \cdot \overline{\operatorname{Grad}} N^{J}\left(\boldsymbol{x}_{\mathrm{r}}^{p}\right)\right] \\
& -\sum_{p=1}^{n_{\text {egp }}} \sum_{q=1}^{n_{\text {egp }}}\left[w^{p} \mathcal{J}\left(\boldsymbol{x}_{\mathrm{r}}^{p}\right) \overline{\operatorname{Grad}} N^{I}\left(\boldsymbol{x}_{\mathrm{r}}^{p}\right) \cdot \mathscr{D}^{\prime}\left(\boldsymbol{x}_{\mathrm{r}}^{p}\right) \boldsymbol{P}_{0}\left(\boldsymbol{x}_{\mathrm{r}}^{p}\right)\right. \\
& \left.\left.\otimes \omega\left(\boldsymbol{x}_{\mathrm{r}}^{p}, \boldsymbol{x}_{\mathrm{s}}^{q}\right) \frac{\partial F_{\mathrm{loc}}\left(\boldsymbol{x}_{\mathrm{s}}^{q}\right)}{\partial \boldsymbol{F}\left(\boldsymbol{x}_{\mathrm{s}}^{q}\right)} \cdot \overline{\operatorname{Grad}} N^{J}\left(\boldsymbol{x}_{\mathrm{s}}^{q}\right)\right]\right],
\end{aligned}
$$

where $\bar{w}, \overline{\mathcal{J}}=\operatorname{det}[\partial \overline{\boldsymbol{x}} / \partial \overline{\boldsymbol{\xi}}]$ and $\bar{n}_{\text {egp }}$ are the interface Gaussian quadrature weights, the interface Jacobian determinant and the number of Gauss points per interface element, respectively. The bulk counterparts of the above parameters are denoted by $w, \mathcal{J}=\operatorname{det}[\partial \boldsymbol{x} / \partial \boldsymbol{\xi}]$ and $n_{\text {egp }}$ respectively. It is also important to define the non-local equivalent distortion Eq. $(15)_{2}$ at a given the same element. The degrees of freedom of the element containing the receiver point $p$ correspond to the rows of the global stiffness matrix whereas the degrees of freedom of the element containing the source point $q$ correspond to the columns of the global stiffness matrix

quadrature point $p$, which reads for the interface

$$
\begin{aligned}
& \bar{F}_{\text {lloc }}\left(\overline{\boldsymbol{x}}_{\mathrm{r}}^{p}\right)=\sum_{q=1}^{\bar{n}_{\mathrm{gp}}} \bar{w}^{q} \overline{\mathcal{J}}\left(\overline{\boldsymbol{x}}_{\mathrm{s}}^{q}\right) \bar{\omega}\left(\overline{\boldsymbol{x}}_{\mathrm{r}}^{p}, \overline{\boldsymbol{x}}_{\mathrm{s}}^{q}\right) \bar{F}_{\mathrm{loc}}\left(\overline{\boldsymbol{x}}_{\mathrm{S}}^{q}\right) \\
& \bar{\omega}\left(\overline{\boldsymbol{x}}_{\mathrm{r}}^{p}, \overline{\boldsymbol{x}}_{\mathrm{s}}^{q}\right)=\frac{\bar{\omega}_{0}\left(\overline{\boldsymbol{x}}_{\mathrm{r}}^{p}, \overline{\boldsymbol{x}}_{\mathrm{s}}^{q}\right)}{\sum_{m=1}^{\bar{n}_{\mathrm{gp}}} \bar{w}^{m} \overline{\mathcal{J}}\left(\overline{\boldsymbol{x}}_{\mathrm{s}}^{m}\right) \bar{\omega}_{0}\left(\overline{\boldsymbol{x}}_{\mathrm{r}}^{p}, \overline{\boldsymbol{x}}_{\mathrm{s}}^{m}\right)},
\end{aligned}
$$

where $\bar{n}_{\mathrm{gp}}$ is the total number of interface Gauss points. Similarly for the bulk, at a given Gauss point $p$ the nonlocal equivalent distortion is defined by

$$
\begin{aligned}
& F_{\mathrm{nloc}}\left(\boldsymbol{x}_{\mathrm{r}}^{p}\right)=\sum_{q=1}^{n_{\mathrm{gp}}} w^{q} \mathcal{J}\left(\boldsymbol{x}_{\mathrm{s}}^{q}\right) \omega\left(\boldsymbol{x}_{\mathrm{r}}^{p}, \boldsymbol{x}_{\mathrm{s}}^{q}\right) F_{\mathrm{loc}}\left(\boldsymbol{x}_{\mathrm{s}}^{q}\right) \\
& \omega\left(\boldsymbol{x}_{\mathrm{r}}^{p}, \boldsymbol{x}_{\mathrm{s}}^{q}\right)=\frac{\omega_{0}\left(\boldsymbol{x}_{\mathrm{r}}^{p}, \boldsymbol{x}_{\mathrm{s}}^{q}\right)}{\sum_{m=1}^{n_{\mathrm{gp}}} w^{m} \mathcal{J}\left(\boldsymbol{x}_{\mathrm{s}}^{m}\right) \omega_{0}\left(\boldsymbol{x}_{\mathrm{r}}^{p}, \boldsymbol{x}_{\mathrm{s}}^{m}\right)},
\end{aligned}
$$
with

where $n_{\mathrm{gp}}$ is the total number of bulk Gauss points. Note that the sums in Eqs. (35) and (36) do not need to be taken over all the Gauss points, but only over those that are located inside the spheres of radii $R$ and $\bar{R}$ centered at Gauss point $p$ (receiver). In order to efficiently calculate the non-local weights $\bar{\omega}\left(\overline{\boldsymbol{x}}_{\mathrm{r}}^{p}, \overline{\boldsymbol{x}}_{\mathrm{s}}^{q}\right)$ for every Gauss point $p$ the following steps are necessary

- find all Gauss points $\overline{\boldsymbol{x}}_{\mathrm{S}}^{q}$ satisfying $\left\|\overline{\boldsymbol{x}}_{\mathrm{r}}^{p}-\overline{\boldsymbol{x}}_{\mathrm{s}}^{q}\right\|<\bar{R}$. Then, 
- evaluate $\bar{\omega}\left(\overline{\boldsymbol{x}}_{\mathrm{r}}^{p}, \overline{\boldsymbol{x}}_{\mathrm{s}}^{q}\right)=\bar{w}^{q} \overline{\mathcal{J}}\left(\overline{\boldsymbol{x}}_{\mathrm{s}}^{q}\right) \bar{\omega}_{0}\left(\left\|\overline{\boldsymbol{x}}_{\mathrm{r}}^{p}-\overline{\boldsymbol{x}}_{\mathrm{s}}^{q}\right\|\right)$,

- compute the sum $\mathcal{S}^{p}=\sum_{q=1}^{\bar{n}_{\mathrm{gp}}} \bar{\omega}\left(\overline{\boldsymbol{x}}_{\mathrm{r}}^{p}, \overline{\boldsymbol{x}}_{\mathrm{s}}^{q}\right)$,

$-\bar{\omega}\left(\overline{\boldsymbol{x}}_{\mathrm{r}}^{p}, \overline{\boldsymbol{x}}_{\mathrm{s}}^{q}\right) \Leftarrow \frac{\bar{\omega}\left(\overline{\boldsymbol{x}}_{\mathrm{r}}^{p}, \overline{\boldsymbol{x}}_{\mathrm{s}}^{q}\right)}{\mathcal{S}^{p}}$,

- store the indices of all Gauss points $q$ associated with the Gauss point $p$ for the assembly.

All of the above steps must be taken once, before the increment loop begins. To find the non-local equivalent distortion it is enough to evaluate $\bar{F}_{\text {nloc }}\left(\overline{\boldsymbol{x}}_{\mathrm{r}}^{p}\right)=$ $\sum_{q=1}^{\bar{n}_{\mathrm{gp}}} \bar{\omega}\left(\overline{\boldsymbol{x}}_{\mathrm{r}}^{p}, \quad \overline{\boldsymbol{x}}_{\mathrm{s}}^{q}\right) \bar{F}_{\mathrm{loc}}\left(\overline{\boldsymbol{x}}_{\mathrm{s}}^{q}\right)$ noting that the factor $\bar{w}^{q} \overline{\mathcal{J}}\left(\overline{\boldsymbol{x}}_{\mathrm{s}}^{q}\right)$ is already included in $\bar{\omega}\left(\overline{\boldsymbol{x}}_{\mathrm{r}}^{p}, \overline{\boldsymbol{x}}_{\mathrm{s}}^{q}\right)$. The similar set of steps are taken as well for the bulk's non-local weights $\omega\left(\boldsymbol{x}_{\mathrm{r}}^{p}, \boldsymbol{x}_{\mathrm{s}}^{q}\right)$.

\subsubsection{Assembly of stiffness matrix}

Some remarks seem necessary when the assembly of the stiffness matrix with non-local contributions is concerned (Jirásek and Patzák 2002). Every pair of Gauss points contributes a small block to the global stiffness matrix with rows corresponding to degrees of freedom of the element containing the point $\overline{\boldsymbol{x}}_{\mathrm{r}}^{p}$ and columns to the degrees of freedom of the element containing the point $\overline{\boldsymbol{x}}_{\mathrm{s}}^{q}$. Therefore the size of this sub-matrix and the corresponding element stiffness matrix are exactly the same as that of the standard nodal and element stiffness matrices. However, the assembly of this block differs in that the global degrees of freedom associated with the rows and columns are not necessarily the same. Every nodal stiffness sub-matrix could be calculated from the pair of nodes that belong to different elements as opposed to the pair of nodes in standard finite element whose elements are the same. The effect of the nonlocality on the structure of the global stiffness matrix is an increased bandwidth and its non-symmetry.

\subsubsection{Algorithmic setup for damage model}

Due to the path-dependence of the damage model, the stress tensor is the solution of a constitutive initial value problem meaning that the stress tensor is not only a function of the instantaneous value of the deformation gradient but also depends on the history of deformation. Therefore an appropriate numerical algorithm for integration of the rate constitutive equations is a requirement in the finite element simulation of such models (de Souza Neto et al. 2011). An example of the integration algorithm is given in Algorithm 2.
In case the damage variable evolves, i.e. step 4 of Algorithm 2, Eq. (17) (or its bulk counterpart) is used to calculate the new value of the damage variable. Once more it is noted that integration Algorithm 2 is written for the interface variables, however, the same algorithm can easily be used for the integration of the bulk's rate variables.

Finally, due to the non-linearity of the constitutive equations, the Newton-Raphson iterative method is used. Algorithm 3 represents this method incorporating Algorithm 2. Algorithm 3 then can be used inside the incremental loop for every load or displacement increment until the final increment $n_{\text {incr }}$ is reached. The incremental non-linear finite element method is depicted in Algorithm 1.

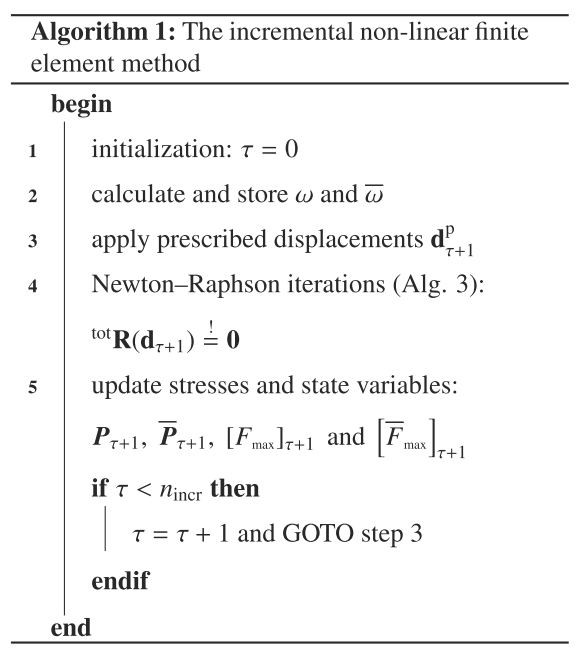

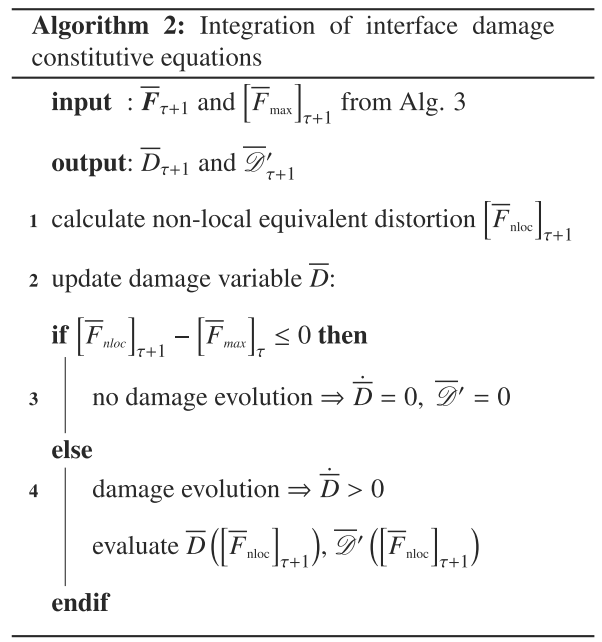




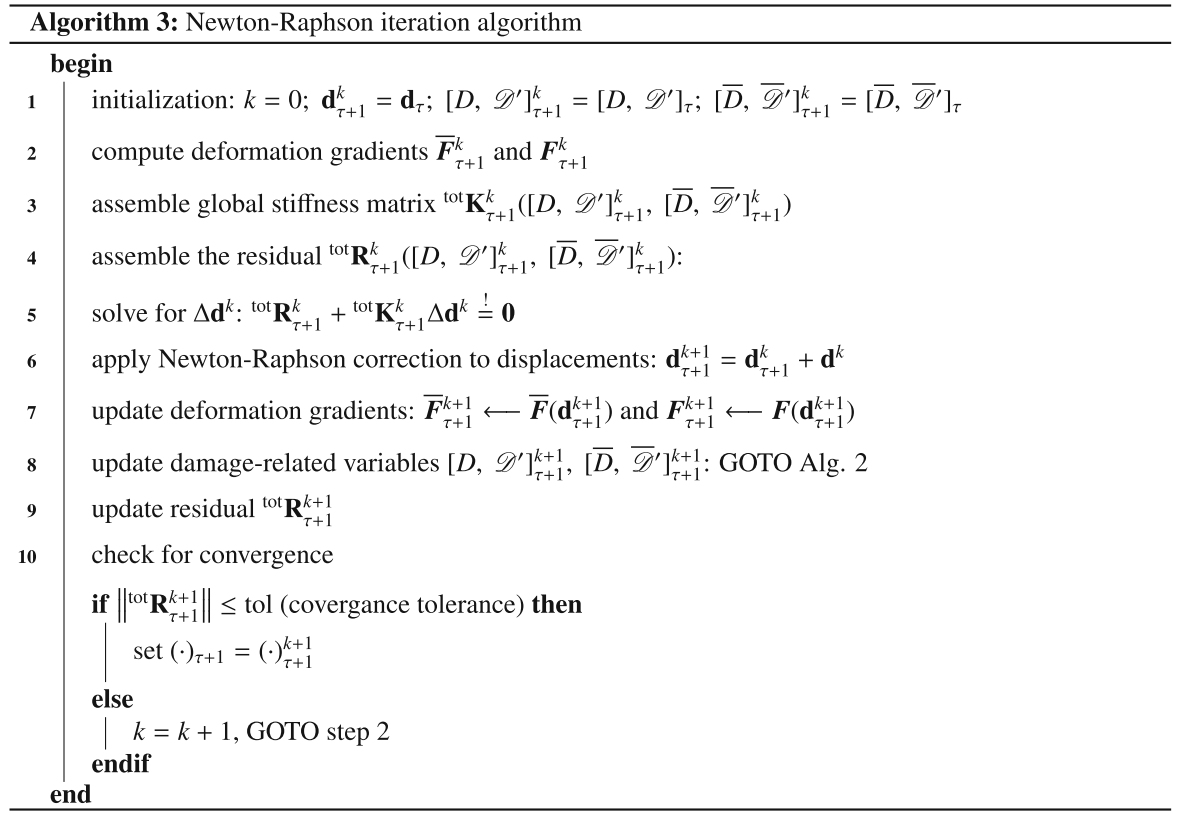

\section{Numerical examples}

The objective of this section is to study the role of an interface (obeying elasticity coupled to non-local damage) on the overall response of the body and to elucidate the theory presented in the previous sections. Three different scenarios are considered and compared to provide a better insight into the proposed model. Also, the effect of the interaction radius on the shape of the damage zone is studied. Unless otherwise stated, the value of the bulk and interface interactive radii $R$ and $\bar{R}$ for all the three cases are 0.015 and $0.03 \mathrm{~mm}$, respectively. It is important to point out that the solution procedure is robust and shows the asymptotic quadratic rate of convergence associated with the Newton-Raphson scheme as expected from the consistently derived (algorithmic) stiffness matrices (see "Appendix 3" for further details). The material behavior in the bulk and on the interface is characterized by hyperelastic Helmholtz energy functions. Table 4 gathers the effective (undamaged) Helmholtz energy functions together with their corresponding derivatives both in the bulk and on the interface (Javili et al. 2013c). The corresponding material parameters for the bulk and interface are given in Table 5. We point out here again that the objectivity of the energies in Table 4 guarantees the symmetry of Cauchy stress and thus a priori satisfies the balance of angular momentum. Moreover, sometimes it is useful to decouple interface deformation into volumetric and isochoric part. The decoupled form of the stress and elasticity tensor are given in "Decoupled form of stress and elasticity tensor" section "Appendix 1".

Remark 4 Fundamental reasoning, atomistic modeling and the Cauchy-Born hypothesis can be employed to construct the interface energy (Fischer et al. 2008; Haiss 2001; Park and Klein 2007). In addition, the surface elastic properties are obtainable utilizing semianalytic methods (Dingreville et al. 2005), ab-initio calculations (Yvonnet et al. 2011b) or atomistic simulation (Davydov et al. 2013). It is also shown by Chatzigeorgiou et al. (2013) that an elastic interface model can be captured by that of an asymptotically zero-thickness bulk. Moreover, the material properties of the interface are independent of the bulk.

Consider the three-dimensional strip shown in Fig. 6. The strip is partitioned into two homogeneous domains by an interface. The width and the thickness of the strip are kept constant. A displacement of $0.002 \mathrm{~mm}$ is prescribed on the two opposite sides, resulting in a constant global loading of the strip. Due to the localized deformations in the damage zones, the resultant deformations are large and require a finite deformation setting. The prescribed displacement is applied in 160 equal steps. The strip is discretized using 10000 trilinear hexahedral elements. For an interactive radius of 
Table 4 Constitutive relations in the bulk and on the interface in the material configuration

\begin{tabular}{ll}
\hline Bulk & Interface \\
\hline$\Psi_{0}(\boldsymbol{F})=\frac{1}{2} \lambda \ln ^{2} J+\frac{1}{2} \mu[\boldsymbol{F}: \boldsymbol{F}-3-2 \ln J]$ & $\bar{\Psi}_{0}(\overline{\boldsymbol{F}})=\frac{1}{2} \bar{\lambda} \ln ^{2} \bar{J}+\frac{1}{2} \bar{\mu}[\overline{\boldsymbol{F}}: \overline{\boldsymbol{F}}-2-2 \ln \bar{J}]$ \\
$\boldsymbol{P}_{0}=\lambda \ln J \boldsymbol{F}^{-\mathrm{t}}+\mu\left[\boldsymbol{F}-\boldsymbol{F}^{-\mathrm{t}}\right]$ & $\overline{\boldsymbol{P}}_{0}=\bar{\lambda} \ln \bar{J} \overline{\boldsymbol{F}}^{-\mathrm{t}}+\bar{\mu}\left[\overline{\boldsymbol{F}}-\overline{\boldsymbol{F}}^{-\mathrm{t}}\right]$ \\
$\mathbb{A}_{0}=\lambda\left[\boldsymbol{F}^{-\mathrm{t}} \otimes \boldsymbol{F}^{-\mathrm{t}}+\ln J \mathbb{D}\right]+\mu[\mathbb{I}-\mathbb{D}]$ & $\overline{\mathbb{A}}_{0}=\bar{\lambda}\left[\overline{\boldsymbol{F}}^{-\mathrm{t}} \otimes \overline{\boldsymbol{F}}^{-\mathrm{t}}+\ln \bar{J} \overline{\mathbb{D}}\right]+\bar{\mu}[\overline{\mathbb{I}}-\overline{\mathbb{D}}]$ \\
$\mathbb{D}=\frac{\partial \boldsymbol{F}^{-\mathrm{t}}}{\partial \boldsymbol{F}}=-\boldsymbol{F}^{-\mathrm{t}} \underline{\otimes} \boldsymbol{F}^{-1}$ & $\overline{\mathbb{D}}=\frac{\partial \overline{\boldsymbol{F}}^{-\mathrm{t}}}{\partial \overline{\boldsymbol{F}}}=-\overline{\boldsymbol{F}}^{-\mathrm{t}} \underline{\boldsymbol{F}}^{-1}+[\boldsymbol{i}-\overline{\boldsymbol{i}}] \bar{\otimes} \overline{\boldsymbol{F}}^{-1} \cdot \overline{\boldsymbol{F}}^{-\mathrm{t}}$ \\
$\mathbb{I}=\frac{\partial \boldsymbol{F}}{\partial \boldsymbol{F}}=\boldsymbol{i} \bar{\otimes} \boldsymbol{I}$ & $\overline{\mathbb{I}}=\frac{\partial \overline{\boldsymbol{F}}}{\partial \overline{\boldsymbol{F}}}=\boldsymbol{i} \bar{\otimes} \overline{\boldsymbol{I}}$ \\
\hline
\end{tabular}

Table 5 Material properties of the numerical examples

\begin{tabular}{lllllll}
\hline & Bulk & \multicolumn{5}{l}{ Interface } \\
\hline Lamé constant & $\mu$ & 80193.8 & $\mathrm{~N} / \mathrm{mm}^{2}$ & $\bar{\mu}$ & $2 \times 80193.8$ & $\mathrm{~N} / \mathrm{mm}$ \\
Lamé constant & $\lambda$ & 110743.5 & $\mathrm{~N} / \mathrm{mm}^{2}$ & $\bar{\lambda}$ & $2 \times 110743.5$ & $\mathrm{~N} / \mathrm{mm}$ \\
Limit elastic strain & $F_{0}$ & 0.003 & & $\bar{F}_{0}$ & 0.0001 & \\
Softening parameter & $F_{\mathrm{f}}$ & 0.1 & & $\bar{F}_{\mathrm{f}}$ & 0.1 & \\
Interaction radius & $R$ & 0.015 & $\mathrm{~mm}$ & $\bar{R}$ & 0.03 & $\mathrm{~mm}$ \\
& $E=\frac{\mu[3 \lambda+2 \mu]}{\mu+\lambda}$ & 206.9 & $\mathrm{~N} / \mathrm{mm}^{2}$ & $\bar{E}=4 \frac{\bar{\mu}[\bar{\lambda}+\bar{\mu}]}{2 \bar{\mu}+\bar{\lambda}}$ & 451.8 & $\mathrm{~N} / \mathrm{mm}$ \\
& & & & & & \\
\hline
\end{tabular}

(a)

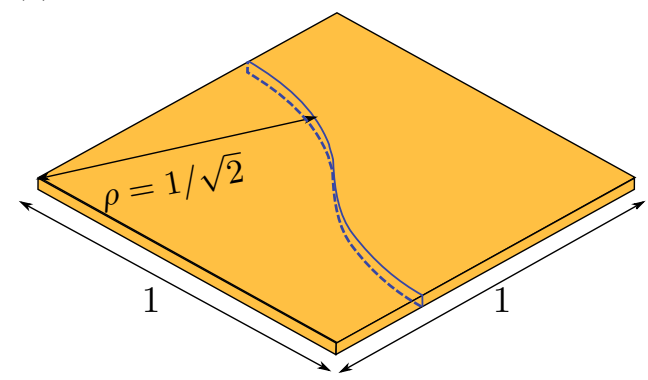

four side surfaces fixed in $y$ direction top \& bottom surfaces fixed in $z$ direction

(b)

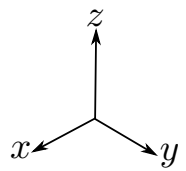

Fig. 6 Strip with curved interface: geometry (a) and applied boundary conditions together with the finite element grid (b). The maximum stretch is $d_{\max }^{\mathrm{p}}=0.004 \mathrm{~mm}$. Dimensions are in $\mathrm{mm}$. The thickness is 0.05

0.010 , the minimum and maximum number of elements within the sphere of non-local influence are 3 and 12 . For an interactive radius of 0.015 , these numbers are 6 and 27, respectively. This increase in the number of influenced elements by the non-locality consequently amplifies the computational efforts. A detailed discussion on this matter is given in "Appendix 3". It is important to point out the values chosen for the bulk and the interface interactive radius $R$ and $\bar{R}$ are here purely numerical. However, we emphasize that these values could be different for the bulk and interface due to the differences in their material structures and scales. Furthermore, identifying the characteristic length scale and relating it to the non-local length scale is still an open discussion (Cordero et al. 2015) and requires extensive experimental tests. For further details see Bažant and 

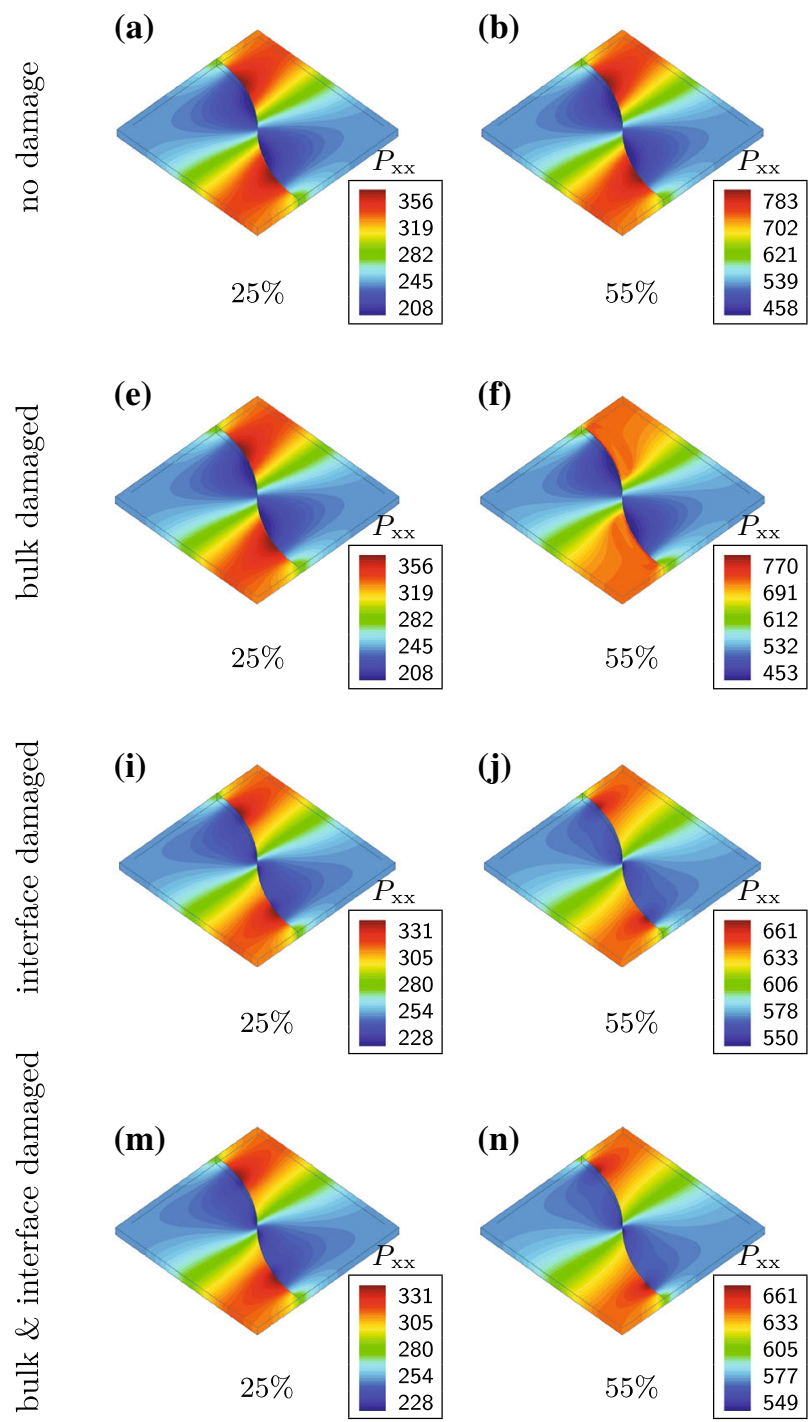

Fig. 7 Bulk stress distributions of the undamaged state of the material and three scenarios for $\bar{\mu} / \mu=\bar{\lambda} / \lambda=2 \mathrm{~mm}$. The results a-d correspond to $25,55,75$, and $100 \%$ of the final deformation $d_{\max }^{\mathrm{p}}$ for the undamaged state of the material, respectively. The results $\mathbf{e}-\mathbf{h}$ correspond to $25,55,75$, and $100 \%$ of the final deformation $d_{\max }^{\mathrm{p}}$ for damaged bulk (case one), respectively. The

Jirásek (2002), Carmeliet (1999), Mazars et al. (1990), Bažant and Cabot (1989).

In the first example we study the mechanical response for damage evolution in the bulk only, in the presence of the interface. The evolution of the stress field is illustrated in Fig. 7e-h. Clearly the stress concentration occurs at points with the highest amount of
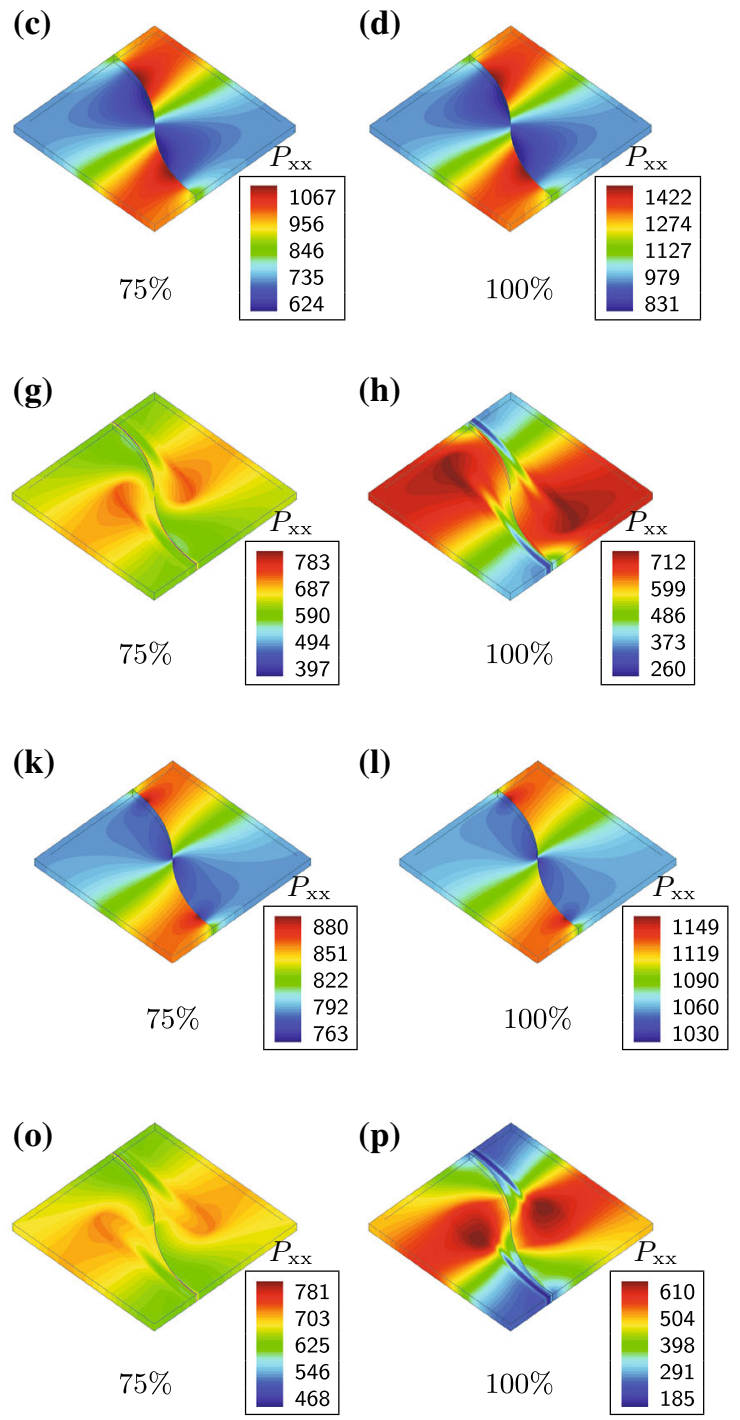

results $\mathbf{i}-\mathbf{l}$ correspond to $25,55,75$, and $100 \%$ of the final deformation $d_{\max }^{\mathrm{p}}$ for damaged interface (case two), respectively. The results $\mathbf{m}-\mathbf{p}$ correspond to $25,55,75$, and $100 \%$ of the final deformation $d_{\max }^{\mathrm{p}}$ for damaged bulk and interface (case three), respectively. The stress depicted is the xx-component of the Piola stress tensor

deformation: adjacent to the portion of the interface with maximum curvature and closest to the boundary on which the displacement is prescribed (see Fig. 7ad). With increasing load, and the onset of damage, the stress concentration zones move with the tip of the damage zone, towards the domain boundary (see Fig. 7f, g). From this point on, the deformation is only localized 


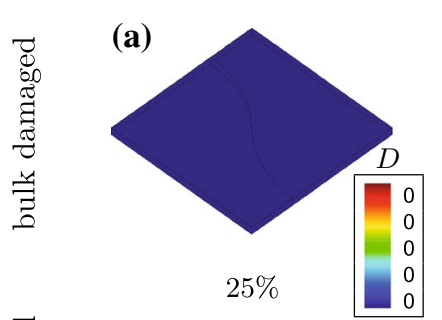

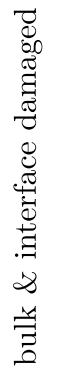
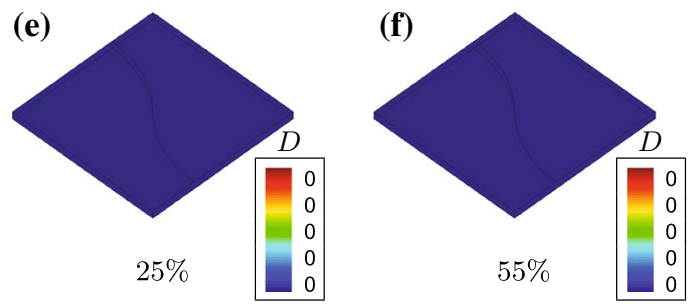

Fig. 8 Bulk damage evolutions of two scenarios for $\bar{\mu} / \mu=$ $\bar{\lambda} / \lambda=2 \mathrm{~mm}$. The results a-d correspond to the bulk damage evolution at $25,55,75$, and $100 \%$ of the final deformation $d_{\max }^{\mathrm{p}}$ for damaged bulk (case one), respectively. The results $\mathbf{e}-\mathbf{h}$ cor-

in the two parallel damage zones, and therefore transmitting lower levels of stress (see Fig. 7h).

The evolution of the damage variable for this case is illustrated in Fig. 8a-d. Note that the damage zone follows closely the interface at first and then, approximately in the middle of the domain, diverges from the interface.

The shape of damage zone strongly depends not only on the interface shape but also on the interactive radius. To study such a dependency an example is devised with a smaller value of the bulk interactive radius $R=0.01 \mathrm{~mm}$ (unlike all the other examples which have $R=0.015 \mathrm{~mm}$ ). The results in Fig. 9a-d are obtained for the first scenario, with $R=0.01 \mathrm{~mm}$ which causes less smooth localization of deformation and that in turn alters the path of the damage propagation. It is also of interest to note that, unlike the damage zones with larger interactive radius, here, the damage zones converge towards the interface.

The second example studies the damage evolution on the interface only and its effects on the overall mechanical response of the body. Figures $7 \mathrm{i}-1$ and $10 \mathrm{e}-\mathrm{h}$ illustrate the stress and damage distribution for this case respectively. The most obvious observation regarding the stress distribution in the bulk, compared
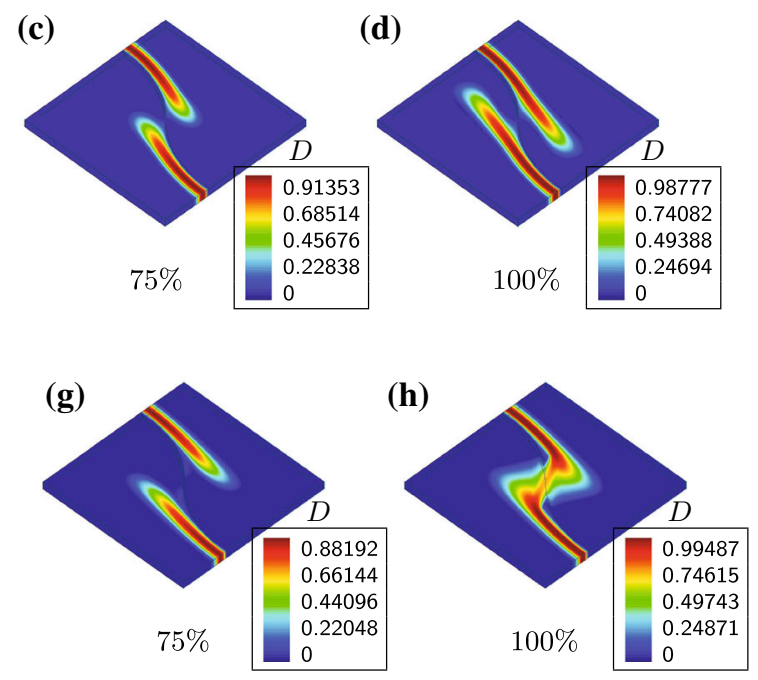

respond to the bulk damage evolution at $25,55,75$, and $100 \%$ of the final deformation $d_{\max }^{\mathrm{p}}$ for damaged bulk and interface (case three), respectively

to case one, is how the stress concentration is now being shifted up- and downwards along the interface while losing its intensity. In addition, the reason for the nonuniform distribution of stress in the bulk is the presence of the energetic interface. Therefore, the more damage the interface develops, the less such a presence is felt in the bulk and the more uniformly the stress is distributed in the domain. This can easily be seen by comparing Fig. $7 \mathrm{~d}$, 1, and noticing the difference between the minimum and maximum values of stresses: the smaller the difference, the less non-uniform the stress distribution. Furthermore, the onset of damage occurs in the middle of the interface and then propagates symmetrically along the interface (Fig. 10e-h) due to the fact that the elements in the middle of the interface undergo the highest level of deformation.

The last case introduces damage both in the bulk and on the interface. The evolution of stress and damage in the bulk are depicted in Figs. $7 \mathrm{~m}-\mathrm{p}$ and $8 \mathrm{e}-\mathrm{h}$. Before the onset of damage in the bulk, the stress distributions in the bulk for case two and three are the same (see Fig. 7i, m for example). However, after the onset of bulk damage, a similar mechanical response to that of case one is observed (see Fig. 7g, o). By increasing the loading, interface damage influences the shape of the 
(a)

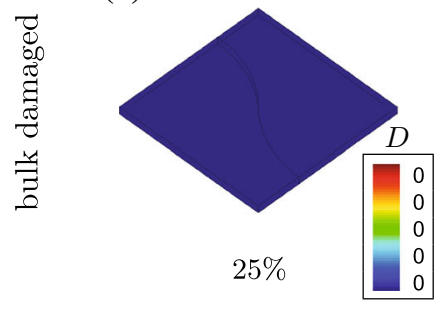

(b)

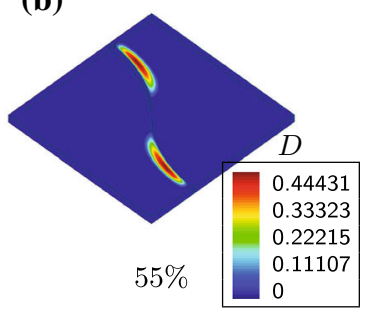

(c)

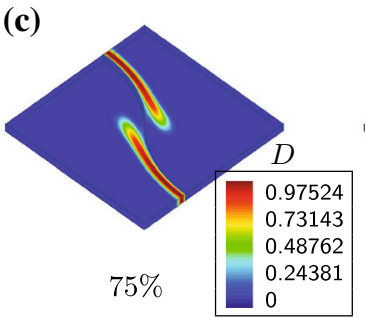

(d)

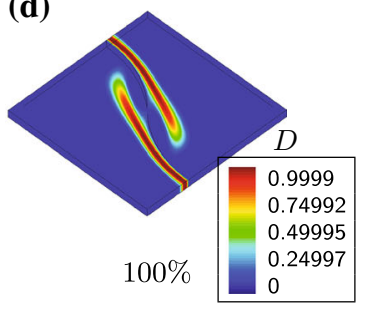

Fig. 9 The effect of smaller interactive radius $R=0.01 \mathrm{~mm}$ on the bulk damage evolution with $\bar{\mu} / \mu=\bar{\lambda} / \lambda=2 \mathrm{~mm}$. The results $\mathbf{a}-\mathbf{d}$ correspond to the bulk damage evolution at 25,55 ,
75 , and $100 \%$ of the final deformation $d_{\max }^{\mathrm{p}}$ for damaged bulk (case one), respectively

increasing deformation

(a)

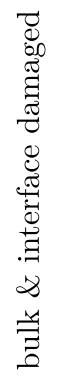

(a)

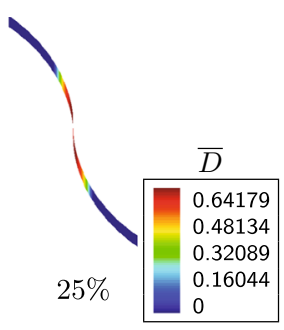

(e)

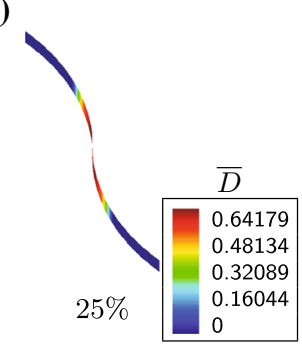

(b)

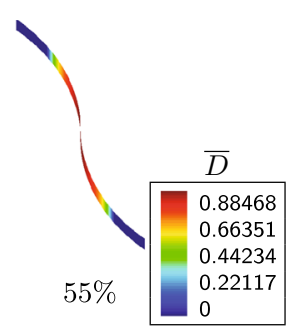

(f)

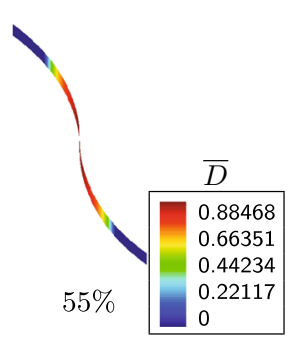

(c)

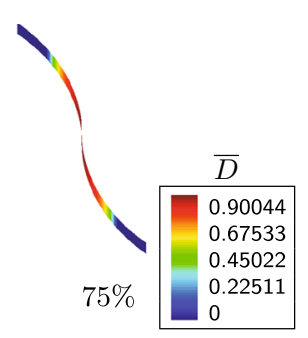

(g)

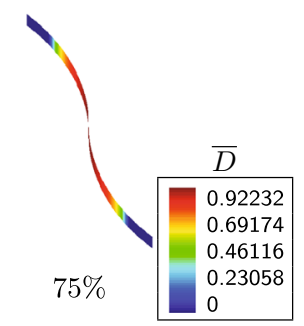

(d)

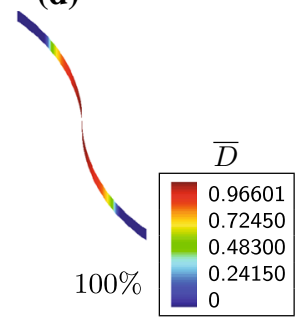

(h)

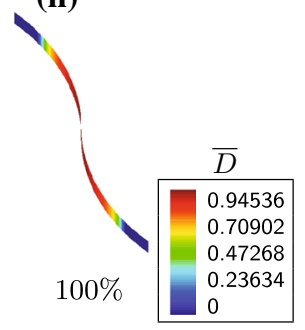

Fig. 10 Interface damage evolutions of two scenarios for $\bar{\mu} / \mu=$ $\bar{\lambda} / \lambda=2 \mathrm{~mm}$. The results $\mathbf{a}-\mathbf{d}$ correspond to the interface damage evolution at $25,55,75$, and $100 \%$ of the final deformation $d_{\max }^{\mathrm{p}}$ for damaged bulk and interface (case three), respectively.
The results $\mathbf{e}-\mathbf{h}$ correspond to the interface damage evolution at $25,55,75$, and $100 \%$ of the final deformation $d_{\max }^{\mathrm{p}}$ for damaged interface (case two), respectively damage zone in the bulk (see Fig. 8c, g) and produces a delay in the onset of damage compared to case one (see Fig. 8b, f). Furthermore, here not only the two damage zones do not diverge from the interface (as in case one, Fig. 8d), they intersect precisely in the middle of the interface, where the interface is damaged the most (see Fig. 8h).

It needs to be emphasized that the delay in the onset of the bulk damage in case three stems from the dam- aged status of the interface. As mentioned before a domain with damaged interface has a more uniform stress distribution and lower stress level which suggest more uniform and smaller local deformations. In other words, bulk elements adjacent to the damaged interface are more relaxed (less deformed) and therefore cause the delay in the onset of the damage.

A comparison of the stress distribution of all the cases at $100 \%$ of the prescribed deformation (see 
increasing deformation

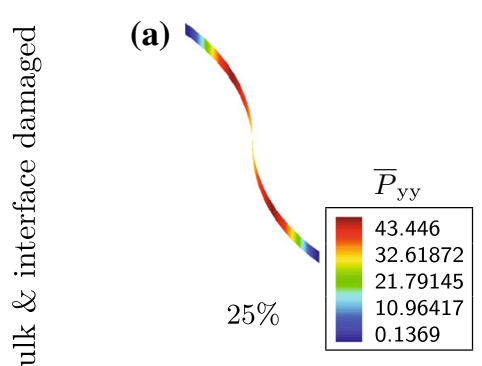

(b)

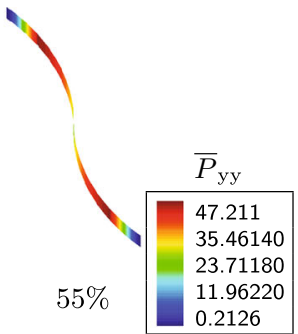

(e)

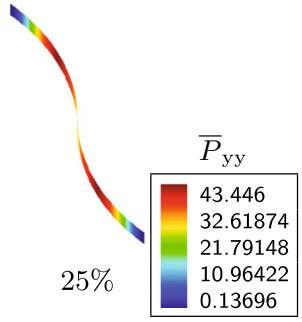

(f)

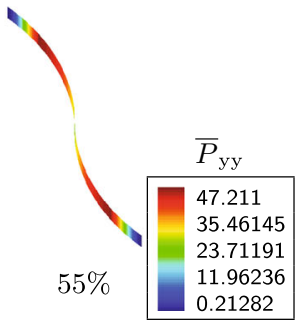

(c)

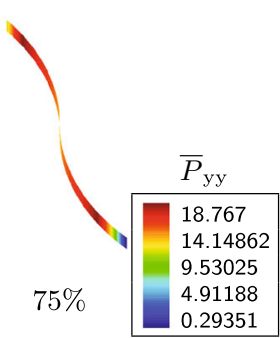

(g)

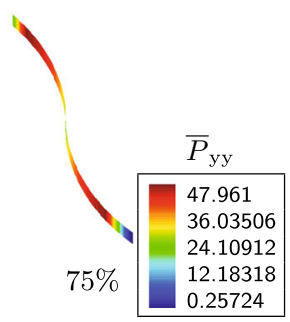

(d)

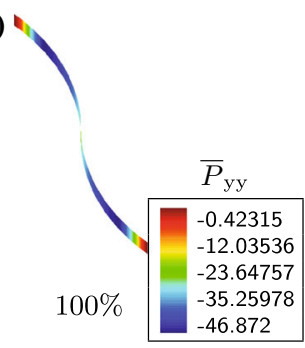

(h)



Fig. 11 Interface stress distributions of three scenarios for $\bar{\mu} / \mu=\bar{\lambda} / \lambda=2 \mathrm{~mm}$. The results a-d correspond to 25,55 , 75 , and $100 \%$ of the final deformation $d_{\max }^{\mathrm{p}}$ for damaged bulk and interface (case three), respectively. The results $\mathbf{e}-\mathbf{h}$ corre-

Fig. $7 \mathrm{~h}, 1, \mathrm{p}$ ) with that of the undamaged material (see Fig. 7d) reveals an overall drop in stress. This drop in case one, two and three is about 50, 20 and $57 \%$. Expectantly the highest drop is associated with case three where both the bulk and interface are damaged. One can also compare the drop in stress levels of case one and three to study the influence of interface damage on the overall response of the body. The interface damage in case three causes about $14 \%$ drop in the overall stress with respect to case one.

The interface stress distribution of case two and three are depicted in Fig. 11a-d and e-h respectively. Up to and including $55 \%$ of the applied loading, both cases have identical results. However, the discrepancy begins with the initiation of the bulk damage in case three resulting in a lower stress level on the interface (see Fig. 11c and g). Such a lower level is achieved in spite of a smaller damage variable of the interface in case three than the one in case two (compare Fig. 10c and g). The reason for this behavior is the damaged neighboring bulk elements transmitting less stress to the interface elements, resulting in the more relaxed interface. spond to $25,55,75$, and $100 \%$ of the final deformation $d_{\max }^{\mathrm{p}}$ for damaged interface (case two), respectively. The stress depicted is the yy-component of the interface Piola stress tensor

The stress evolution of two nodes, one in the bulk and one on the interface (see Fig. 6b) are illustrated in Fig. 12a and $b^{5}$ respectively. One can observe the reduction in the stress for case one (only bulk damaged) and three (both bulk and interface damaged) measured at the interface (-on-interfaceNode) and bulk (on-bulkNode) node from the onset of damage on, representing the decay of the stiffness of the material (see Fig. 12a). Furthermore for case two (only interface damaged) clearly no softening in the bulk is seen.

The interface stress evolutions of all the cases measured at the interface node, exhibit more distinct behavior (see Fig. 12b). When damage exists only in the bulk (case one), the interface stress reduction is the direct result of stress reduction in the bulk. For the second

\footnotetext{
5 A remark on the legends of the graphs is necessary. The legends "case 1", "case2" and "case 3" represent the locations where damage initiates and evolves, which are in the bulk, on the interface and in both the bulk and interface respectively. The "-oninterfaceNode" and "on-bulkNode" part of all the legends stand for the location of the nodes on which the measurements are done to draw the graphs (see Fig. 6). The "-on-interfaceNode " $_{b}$ means a bulk quantity is measured at the interface node.
} 
(a)

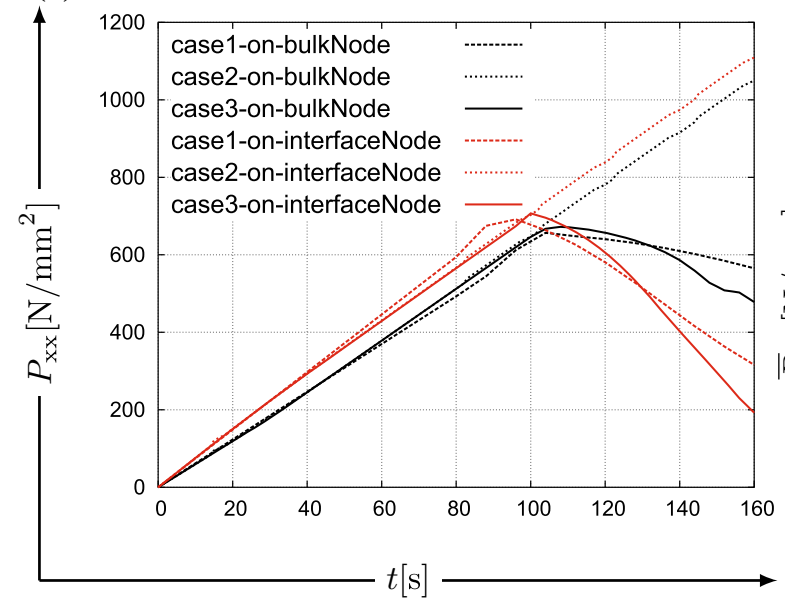

Fig. 12 Bulk (a) and interface (b) Piola stress evolutions of all the three scenarios; case one: only bulk damaged, case two: only interface damaged and case three: both bulk and inter- (b)

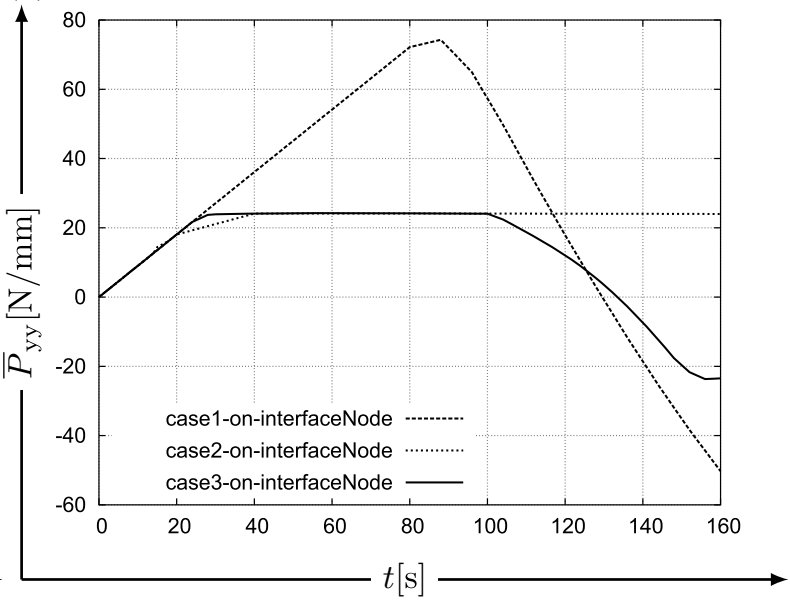

face damaged, measured at two nodes: one in the bulk (onbulkNode) and one on the interface (on-interfaceNode) with $\bar{\mu} / \mu=\bar{\lambda} / \lambda=2 \mathrm{~mm}$ (a)

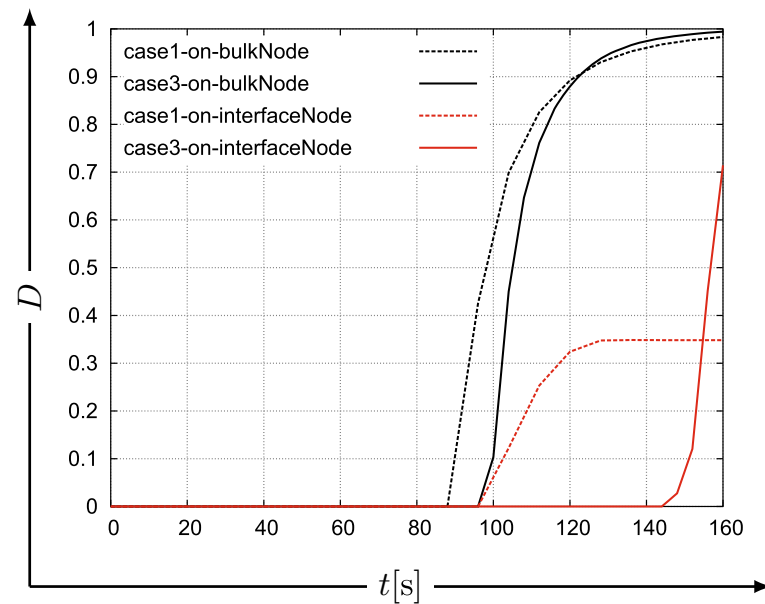

Fig. 13 Bulk (a) and interface (b) damage variable evolutions of all the applicable scenarios; case one: only bulk damaged, case two: only interface damaged and case three: both bulk

case (interface damaged), one can observe a very gradual decrease of stress, due to a gradual increase of interface damage under increasing loading. In case three (both bulk and interface damaged), since the onset of interface damage is faster than that of the bulk, a similar stress behavior to that of case two (interface damaged) is observed. However, with the onset of the damage in the bulk (around step 100) a similar response to that of case one (bulk damaged) is seen. (b)

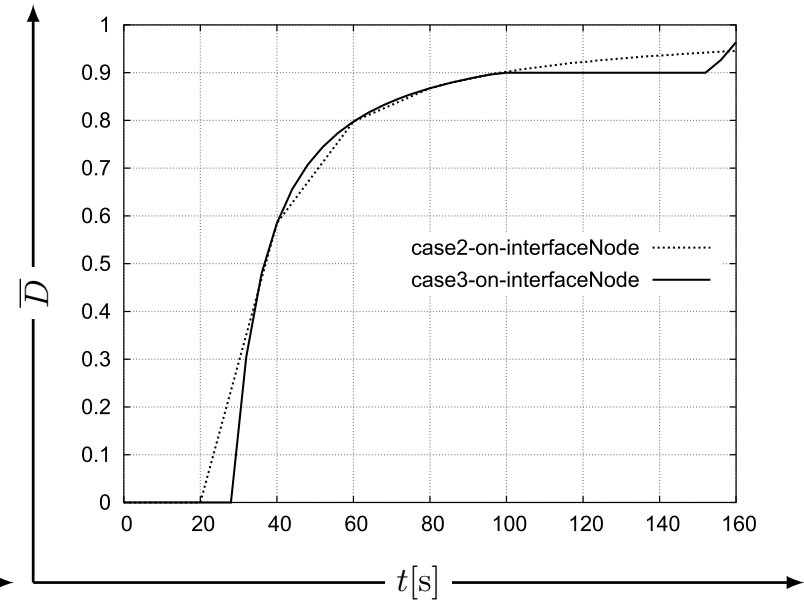

and interface damaged, measured at two nodes: one in the bulk (on-bulkNode) and one on the interface (on-interfaceNode) with $\bar{\mu} / \mu=\bar{\lambda} / \lambda=2 \mathrm{~mm}$

The evolution of the damage variable $D$ and the nonlocal equivalent distortion $F_{\text {nloc }}$ in the bulk are gradual and constantly increasing (black curves in Figs. 13a, 14a). However, for the evolution of the same parameters measured at the interface node a more dramatic change specially at higher levels of loading is observed for case three (red curves in Figs. 13a, 14b which is in agreement with the fact that two damage zones intersect at this location increasing the local deformation and damage. 
(a)

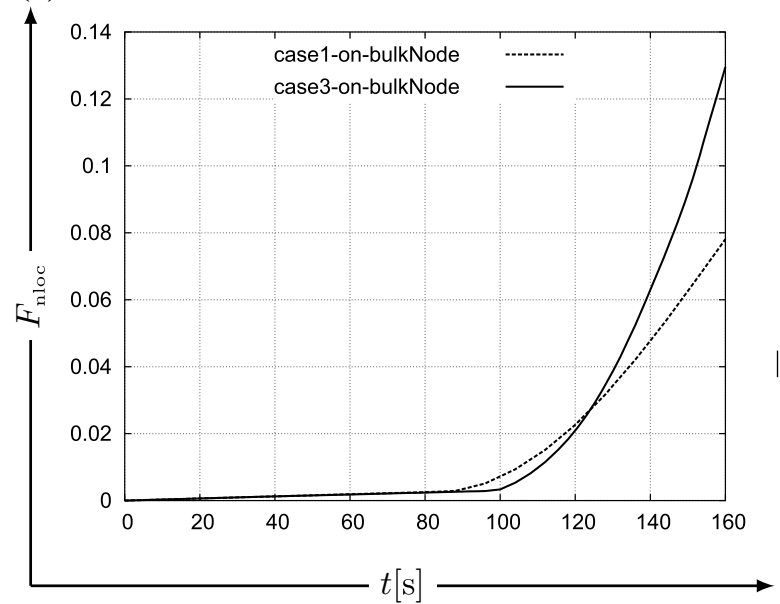

Fig. 14 Only bulk (a) and both interface and bulk (b) equivalent deformation distortion of all the applicable case scenarios; case one: only bulk damaged, case two: only interface damaged and case three: both bulk and interface damaged, measured at two nodes: one in the bulk (on-bulkNode) and one on the inter-

Additionally one can observe in Fig. 14a that although the prescribed displacement is only $0.4 \%$ of the domain length in the direction of applied displacement $(1 \mathrm{~mm})$, locally a deformation as high as $14 \%$ is achieved which is why a finite deformation formulation is needed and implemented.

Finally the black curves in Fig. 14b, the evolutions of the interface equivalent distortion for case two and three, reveal an important mechanical behavior. When only the interface is damaged, a gradual increase in equivalent distortion is observed which in turn results in the gradual increase of the damage variable (see dotted curve in Fig. 13b). This gradual increase is not obtained for case three (see solid curve in Fig. 13b) since around step 100 with the onset of bulk damage, the evolution of the interface damage is discontinued. This behavior persists up until approximately step 150 . This interval (step 100-150) corresponds to the unloading-reloading segment of the solid black curve in Fig. 14b. However, for the last 10 steps with the non-local equivalent distortion continuously reaching new maximum values, the interface damage variable continues to evolve.

\section{Summary and conclusion}

A three-dimensional finite element framework for continua containing elastic coherent interfaces subject to damage is presented. The interface is endowed with (b)

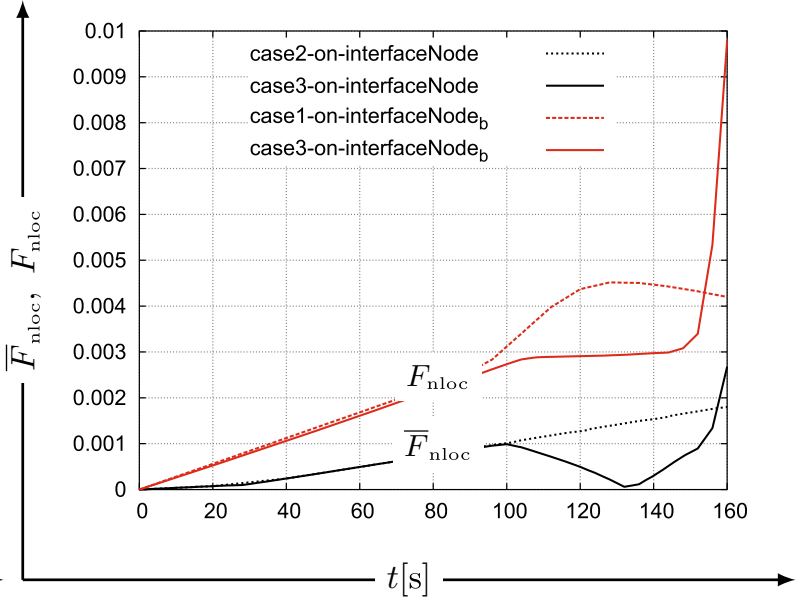

face (on-interfaceNode) with $\bar{\mu} / \mu=\bar{\lambda} / \lambda=2 \mathrm{~mm}$. In $\mathbf{b}$, the graphs of case 1 and case 3 with the measurement location of oninterfaceNode $\mathrm{b}_{\mathrm{b}}$ are drawn for a bulk quantity measured at the interface node

its own mechanically energetic structure. A class of integral-type non-local isotropic damage models is employed and coupled with the current framework and implemented for both the bulk and the interface. The non-locality is applied to the equivalent distortion. The corresponding weak forms of the balance equation including the contributions from the interface are given. The balance equations are fully discretized using the finite element method. A material model for the interface Helmholtz energy is then introduced. The exact consistent stiffness matrix in the bulk and on the interface is derived taking the non-local damage contributions into consideration. The non-locality affects the symmetry and bandwidth of the stiffness matrix. To minimize the number of nonzero elements in the stiffness matrix a bell-shaped weighting function is used to calculate the weighting coefficients needed to nonlocalize the local equivalent distortion. To account for the boundary effects typical for non-local damage models, the weight functions are normalized. In addition these weight functions (for the bulk and interface) are computed on the undeformed configuration for reasons of objectivity.

A series of three-dimensional numerical examples serves to elucidate the role of interface elasticity on the overall response of the body (with and without damage). It is shown that interface elasticity alone influences the shape of the damage zone in the bulk by 
(a)

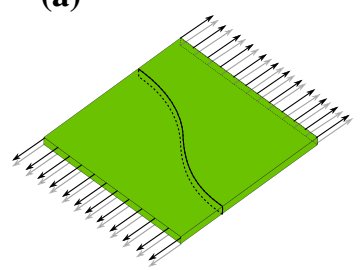

(b)
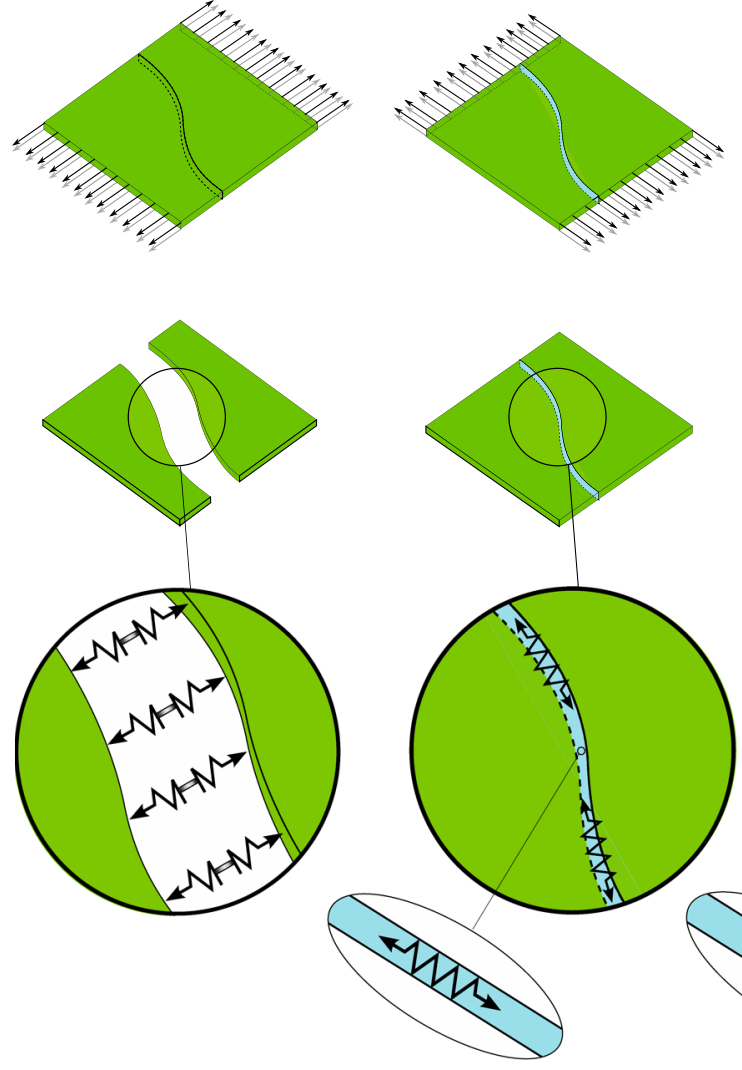

damage type

(cohesive) interface model (c)
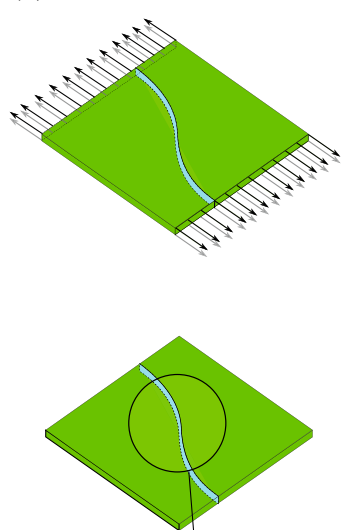

(d)
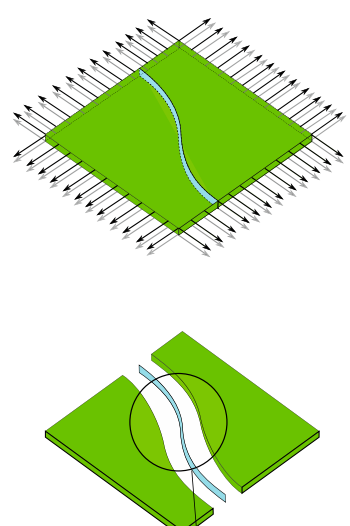

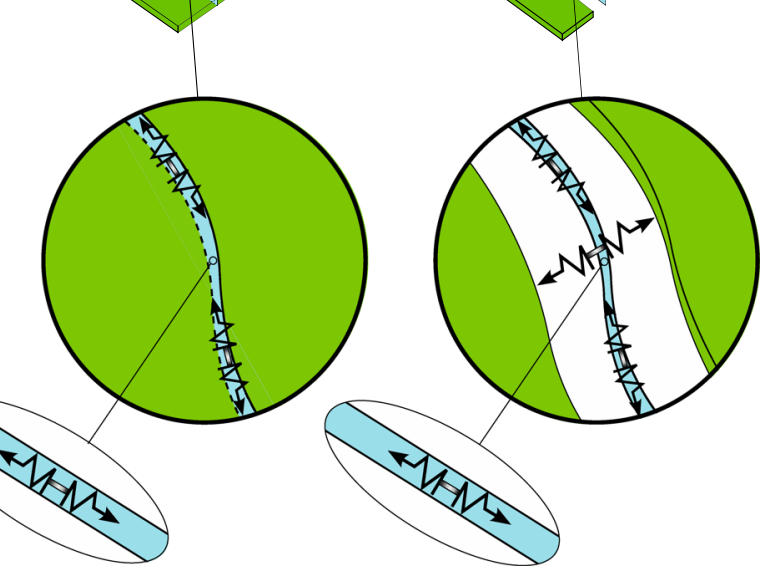

interface elasticity with damage interface elasticity with damage coherent interfaces current work

\begin{abstract}
non-coherent interfaces
\end{abstract} future contribution

elasticity theory together with continuum damage (current contribution); d loadings "parallel" and "normal" to the interface causing both opening across and in-plane deformation of the interface, accounting for interface damage, modeled by interface elasticity theory together with cohesive zone and continuum damage (the future work). The different color of the interface represents different interface material properties as compared to those of the bulk

active radius results in a different shape and path of the damage zone and therefore care needs to be taken when choosing the interactive radius.

The current contribution extends the classical interface elasticity theory Fig. 15b to account for in-plane degradation of the interface Fig. 15c. This framework is limited only to coherent interfaces. The further extension of this work to non-coherent interfaces shall be elaborated in a future contribution in which a traction-separation law similar to that of the cohesive zone model Fig. 15a is assumed to relate the 
interface traction to the displacement jump across the interface Fig. 15d. In addition, it is straightforward to employ more sophisticated damage mechanisms such as anisotropic damage models. The treatment of nonlocal quantities in the vicinity of the boundary also requires some modifications according to the works of Krayani et al. (2009), Pijaudier-Cabot and Dufour (2010). A study is also necessary on the interactive radius, a parameter that is usually determined by experiments. Moreover an investigation of the influences of the bulk and interface inelasticity on the thermomechanical responses of the body is of great importance. These extensions shall be discussed in later contributions.

Acknowledgements This research is performed as part of the Energie Campus Nuremberg and supported by funding through the "Bavaria on the Move" initiative of the state of Bavaria. The authors also gratefully acknowledge the support by the Cluster of Excellence "Engineering of Advanced Materials".

\section{Appendix 1: Some mathematical relations and derivations}

In this section we present the derivation of the balance of forces on the interface and the corresponding weak form. Subsequently, the decoupled form of the interface energy, Piola stress and elasticity tensor are provided. Some useful identities and relations used in the derivations are also given without proof.

\section{Extended divergence theorem}

The extended forms of divergence theorem in the material configuration for a bulk tensor field $\{\bullet\}$ and a tensorial quantity on the interface $\{\overline{\boldsymbol{\theta}}\}$ are

$$
\begin{aligned}
& \int_{\mathcal{B}_{0}} \operatorname{Div}\{\bullet\} \mathrm{d} V=\int_{\partial \mathcal{B}_{0}}\{\bullet\} \cdot N \mathrm{~d} A-\int_{\mathcal{I}_{0}} \llbracket \bullet \rrbracket \cdot \overline{\boldsymbol{N}} \mathrm{d} A \\
& \int_{\mathcal{I}_{0}} \overline{\operatorname{Div}}\{\bar{\bullet}\} \mathrm{d} A=\int_{\partial \mathcal{I}_{0} \backslash \partial \mathcal{I}_{0}^{\mathrm{N}}}\{\bar{\bullet}\} \cdot \widetilde{\boldsymbol{N}} \mathrm{d} L \\
& \quad+\int_{\partial \mathcal{I}_{0}^{\mathrm{N}}}\{\bar{\bullet}\} \cdot \widetilde{\boldsymbol{N}} \mathrm{d} L-\int_{\mathcal{I}_{0}} \bar{C}\{\bar{\bullet}\} \cdot \overline{\boldsymbol{N}} \mathrm{d} A
\end{aligned}
$$

where the curvature of the interface is denoted by $\bar{C}$. Note that $\partial \mathcal{I}_{0}^{\mathrm{N}}$ is the portion of the interface boundary that intersects with the bulk's boundary, thus $\partial \mathcal{I}_{0} \backslash$ $\partial \mathcal{I}_{0}^{\mathrm{N}} \cap \partial \mathcal{B}_{0}=\emptyset$.
Balance of forces on interface

The global form of the balance of forces both in the bulk and on the interface is given as (see, Javili and Steinmann 2010b, for further details concerning thermomechanical solids with surface energy only)

$$
\begin{gathered}
\int_{\mathcal{B}_{0}} \boldsymbol{B}^{\mathrm{p}} \mathrm{d} V+\int_{\mathcal{I}_{0}} \overline{\boldsymbol{B}}^{\mathrm{p}} \mathrm{d} A+\int_{\partial \mathcal{B}_{0}^{\mathrm{N}}} \widehat{\boldsymbol{B}}_{\mathrm{N}}^{\mathrm{p}} \mathrm{d} A+\int_{\partial \mathcal{I}_{0}^{\mathrm{N}}} \widetilde{\boldsymbol{B}}_{\mathrm{N}}^{\mathrm{p}} \mathrm{d} L \\
+\int_{\partial \mathcal{B}_{0} \backslash \partial \mathcal{B}_{0}^{\mathrm{N}}} \boldsymbol{P} \cdot \boldsymbol{N} \mathrm{d} A+\int_{\partial \mathcal{I}_{0} \backslash \partial \mathcal{I}_{0}^{\mathrm{N}}} \overline{\boldsymbol{P}} \cdot \widetilde{\boldsymbol{N}} \mathrm{d} L=\mathbf{0} .
\end{gathered}
$$

Taking the limit $\mathcal{B}_{0} \rightarrow \emptyset$, and consequently $\partial \mathcal{B}_{0}=\mathcal{I}_{0}$, with $\boldsymbol{N}=\overline{\boldsymbol{N}}$ on $\mathcal{I}_{0}^{+}, \boldsymbol{N}=-\overline{\boldsymbol{N}}$ on $\mathcal{I}_{0}^{-}, \partial \mathcal{I}_{0}^{\mathrm{N}}=\emptyset$, $\partial \mathcal{B}_{0}^{\mathrm{N}}=\varnothing$, and taking into account the extended forms of the divergence theorem (37) and (38), one obtains the local balance of forces on the interface as

$$
\begin{gathered}
\int_{\partial \mathcal{I}_{0}} \overline{\boldsymbol{P}} \cdot \widetilde{\boldsymbol{N}} \mathrm{d} L+\int_{\mathcal{I}_{0}} \boldsymbol{P} \cdot \boldsymbol{N} \mathrm{d} A+\int_{\mathcal{I}_{0}} \overline{\boldsymbol{B}}^{\mathrm{p}} \mathrm{d} A=\mathbf{0} \\
\Longrightarrow \int_{\mathcal{I}_{0}} \overline{\operatorname{Div}} \overline{\boldsymbol{P}}+\llbracket \boldsymbol{P} \rrbracket \cdot \overline{\boldsymbol{N}}+\overline{\boldsymbol{B}}^{\mathrm{p}} \mathrm{d} A=\mathbf{0} .
\end{gathered}
$$

From arbitrariness of $\mathcal{B}_{0}$ and thus $\mathcal{I}_{0}$, the balance of force on the interface listed in Table 2 then follows. In the case that the interface is not energetic i.e. $\overline{\boldsymbol{P}}=\mathbf{0}$, and in the absence of interface body force $\left(\overline{\boldsymbol{B}}^{\mathrm{p}}=\mathbf{0}\right)$, the classical traction continuity condition is recovered.

Weak form of the balance of forces

The localized balance equations in the bulk and on the interface, given in Table 2 are tested from the left with vector valued functions $\delta \boldsymbol{\varphi}$ and $\delta \bar{\varphi}$, respectively as follows

$$
\begin{aligned}
& \int_{\mathcal{B}_{0}} \delta \boldsymbol{\varphi} \cdot\left[\operatorname{Div} \boldsymbol{P}+\boldsymbol{B}^{\mathrm{p}}\right] \mathrm{d} V \\
& \quad+\int_{\mathcal{I}_{0}} \delta \overline{\boldsymbol{\varphi}} \cdot\left[\overline{\operatorname{Div}} \overline{\boldsymbol{P}}+\overline{\boldsymbol{B}}^{\mathrm{p}}+\llbracket \boldsymbol{P} \rrbracket \cdot \overline{\boldsymbol{N}}\right] \mathrm{d} A=0,
\end{aligned}
$$

which can be alternatively written as

$$
\begin{aligned}
& \int_{\mathcal{B}_{0}}-\boldsymbol{P}: \operatorname{Grad} \delta \boldsymbol{\varphi}+\operatorname{Div}(\delta \boldsymbol{\varphi} \cdot \boldsymbol{P})+\delta \boldsymbol{\varphi} \cdot \boldsymbol{B}^{\mathrm{p}} \mathrm{d} V \\
& \quad+\int_{\mathcal{I}_{0}}-\overline{\boldsymbol{P}}: \overline{\operatorname{Grad}} \delta \overline{\boldsymbol{\varphi}}+\overline{\operatorname{Div}}(\delta \overline{\boldsymbol{\varphi}} \cdot \overline{\boldsymbol{P}}) \mathrm{d} A \\
& \quad+\int_{\mathcal{I}_{0}} \delta \overline{\boldsymbol{\varphi}} \cdot \overline{\boldsymbol{B}}^{\mathrm{p}}+\delta \overline{\boldsymbol{\varphi}} \cdot[\llbracket \boldsymbol{P} \rrbracket \cdot \overline{\boldsymbol{N}}] \mathrm{d} A=0,
\end{aligned}
$$


and using the extended forms of divergence theorem (37) and (38), for various parts of the body results in

$$
\begin{aligned}
& \int_{\mathcal{B}_{0}} \boldsymbol{P}: \operatorname{Grad} \delta \boldsymbol{\varphi} \mathrm{d} V-\int_{\partial \mathcal{B}_{0}^{\mathrm{N}}} \delta \boldsymbol{\varphi} \cdot[\boldsymbol{P} \cdot \boldsymbol{N}] \mathrm{d} A \\
& +\int_{\mathcal{I}_{0}} \llbracket \delta \boldsymbol{\varphi} \cdot \boldsymbol{P} \rrbracket \cdot \overline{\boldsymbol{N}} \mathrm{d} A-\int_{\mathcal{B}_{0}} \delta \boldsymbol{\varphi} \cdot \boldsymbol{B}^{\mathrm{p}} \mathrm{d} V \\
& +\int_{\mathcal{I}_{0}} \overline{\boldsymbol{P}}: \overline{\operatorname{Grad}} \delta \overline{\boldsymbol{\varphi}} \mathrm{d} A-\int_{\partial \mathcal{I}_{0}^{\mathrm{N}}} \delta \overline{\boldsymbol{\varphi}} \cdot[\overline{\boldsymbol{P}} \cdot \tilde{\boldsymbol{N}}] \mathrm{d} L \\
& -\int_{\mathcal{I}_{0}} \delta \overline{\boldsymbol{\varphi}} \cdot \overline{\boldsymbol{B}}^{\mathrm{p}}+\delta \overline{\boldsymbol{\varphi}} \cdot[\llbracket \boldsymbol{P} \rrbracket \cdot \overline{\boldsymbol{N}}] \mathrm{d} A=0 .
\end{aligned}
$$

On the Neumann boundaries of the bulk and interface, $\boldsymbol{P} \cdot \boldsymbol{N}=\widehat{\boldsymbol{B}}_{\mathrm{N}}^{\mathrm{p}}$ and $\overline{\boldsymbol{P}} \cdot \widetilde{\boldsymbol{N}}=\widetilde{\boldsymbol{B}}_{\mathrm{N}}^{\mathrm{p}}$, respectively. Noting $\llbracket \delta \boldsymbol{\varphi} \cdot \boldsymbol{P} \rrbracket=\llbracket \delta \boldsymbol{\varphi} \rrbracket \cdot\{\boldsymbol{P}\}+\llbracket \delta \delta \boldsymbol{\varphi}\} \cdot \llbracket \boldsymbol{P} \rrbracket, \llbracket \delta \varphi \rrbracket=\mathbf{0}$, for coherent interfaces, and $\{\delta \boldsymbol{\varphi}\}=\delta \overline{\boldsymbol{\varphi}}$, Eq. (43) simplifies to the weak form Eq. (18).

\section{Decoupled form of stress and elasticity tensor}

It is sometimes useful to decouple the bulk deformation into the volumetric and isochoric part. In analogy, the volumetric $^{6}$ and isochoric part of the interface deformation read

$\overline{\boldsymbol{F}}=\overline{\boldsymbol{F}}^{\mathrm{vol}} \overline{\boldsymbol{F}}^{\text {iso }} \quad$ with $\quad \overline{\boldsymbol{F}}^{\mathrm{vol}}=\overline{\boldsymbol{J}}^{1 / 2} \overline{\boldsymbol{I}}$

$$
\text { and } \overline{\boldsymbol{F}}^{\text {iso }}=\bar{J}^{-1 / 2} \overline{\boldsymbol{F}} \text {. }
$$

Furthermore, the Helmholtz energy can be written as

$\bar{\Psi}(\overline{\boldsymbol{F}})=\bar{\Psi}\left(\overline{\boldsymbol{F}}^{\mathrm{iso}}, \overline{\boldsymbol{F}}^{\mathrm{vol}}\right)$

The Piola stress reads

$$
\begin{aligned}
& \overline{\boldsymbol{P}}:=\frac{\partial \bar{\Psi}}{\partial \overline{\boldsymbol{F}}}=\frac{\partial \bar{\Psi}}{\partial \overline{\boldsymbol{F}}^{\mathrm{iso}}}: \frac{\partial \overline{\boldsymbol{F}}^{\mathrm{iso}}}{\partial \overline{\boldsymbol{F}}}+\frac{\partial \bar{\Psi}}{\partial \overline{\boldsymbol{F}}^{\mathrm{vol}}}: \frac{\partial \overline{\boldsymbol{F}}_{\mathrm{vol}}}{\partial \overline{\boldsymbol{F}}} \text { or } \\
& \overline{\boldsymbol{P}}=\overline{\boldsymbol{P}}^{\mathrm{iso}}+\overline{\boldsymbol{P}}^{\mathrm{vol}},
\end{aligned}
$$

with

$$
\begin{aligned}
& \overline{\boldsymbol{P}}^{\mathrm{vol}}=\frac{\partial \bar{\Psi}}{\partial \overline{\boldsymbol{F}}^{\mathrm{vol}}}: \frac{\partial \overline{\boldsymbol{F}}^{\mathrm{vol}}}{\partial \overline{\boldsymbol{F}}}=\frac{\partial \bar{\Psi}}{\partial \overline{\boldsymbol{F}}^{\mathrm{vol}}}:\left[\frac{1}{2} \bar{J}^{1 / 2} \overline{\boldsymbol{I}} \otimes \overline{\boldsymbol{F}}^{-\mathrm{t}}\right], \\
& \overline{\boldsymbol{P}}^{\text {iso }}=\frac{\partial \bar{\Psi}}{\partial \overline{\boldsymbol{F}}^{\text {iso }}}: \frac{\partial \overline{\boldsymbol{F}}^{\text {iso }}}{\partial \overline{\boldsymbol{F}}}=\bar{J}^{-1 / 2} \frac{\partial \bar{\Psi}}{\partial \overline{\boldsymbol{F}}^{\text {iso }}}:\left[\overline{\mathbb{I}}-\frac{1}{2} \overline{\boldsymbol{F}} \otimes \overline{\boldsymbol{F}}^{-\mathrm{t}}\right] .
\end{aligned}
$$

\footnotetext{
6 The term "volumetric" has a different meaning on the interface. As opposed to a volumetric deformation in the bulk, which changes the volume uniformly, a volumetric interface deformation changes the area uniformly.
}

The Piola stress tangent follows

$$
\overline{\mathbb{A}}:=\frac{\partial \overline{\boldsymbol{P}}}{\partial \overline{\boldsymbol{F}}}=\frac{\partial \overline{\boldsymbol{P}}^{\text {iso }}}{\partial \overline{\boldsymbol{F}}}+\frac{\partial \overline{\boldsymbol{P}}^{\mathrm{vol}}}{\partial \overline{\boldsymbol{F}}} \quad \text { or } \quad \overline{\mathbb{A}}=\overline{\mathbb{A}}^{\text {iso }}+\overline{\mathbb{A}}^{\mathrm{vol}},
$$

with

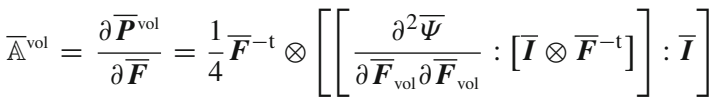

$$
\begin{aligned}
& -\frac{1}{4} \overline{\boldsymbol{F}}^{-\mathrm{t}} \otimes\left[\bar{J}^{-1 / 2} \frac{\partial \bar{\Psi}}{\partial \overline{\boldsymbol{F}}_{\mathrm{vol}}}:\left[\overline{\boldsymbol{I}} \otimes \overline{\boldsymbol{F}}^{-\mathrm{t}}\right]\right] \\
& +\frac{1}{2} \bar{J}^{-1 / 2}\left[\frac{\partial \bar{\Psi}}{\partial \overline{\boldsymbol{F}}_{\mathrm{vol}}}: \overline{\boldsymbol{I}}\right] \overline{\mathbb{D}}, \\
& \overline{\mathbb{A}}^{\text {iso }}=\frac{\partial \overline{\boldsymbol{P}}^{\text {iso }}}{\partial \overline{\boldsymbol{F}}}=-\frac{1}{2} \bar{J}^{-1 / 2}\left[\frac{\partial \bar{\Psi}}{\partial \overline{\boldsymbol{F}}^{\text {iso }}}:\left[\overline{\mathbb{I}}-\frac{1}{2} \overline{\boldsymbol{F}} \otimes \overline{\boldsymbol{F}}^{-\mathrm{t}}\right]\right] \otimes \overline{\boldsymbol{F}}^{-\mathrm{t}} \\
& +\bar{J}^{-1 / 2}\left[\overline{\mathbb{A}}_{1}-\frac{1}{2}\left[\overline{\mathbb{A}}_{2}+\overline{\mathbb{A}}_{3}\right]\right],
\end{aligned}
$$

where

$$
\begin{aligned}
& \overline{\mathbb{A}}_{1}=[\overline{\boldsymbol{I}} \bar{\otimes} \boldsymbol{i}]: \frac{\partial^{2} \bar{\Psi}}{\partial \overline{\boldsymbol{F}}^{\text {is }} \partial \overline{\boldsymbol{F}}^{\text {iso }}}:\left[\overline{\mathbb{I}}-\frac{1}{2} \overline{\boldsymbol{F}} \otimes \overline{\boldsymbol{F}}^{-\mathrm{t}}\right], \\
& \overline{\mathbb{A}}_{2}=\left[\frac{\partial \bar{\Psi}}{\partial \overline{\boldsymbol{F}}^{\text {iso }}}: \overline{\boldsymbol{F}}\right] \overline{\mathbb{D}}, \\
& \overline{\mathbb{A}}_{3}=\overline{\boldsymbol{F}}^{-\mathrm{t}} \otimes\left[\frac{\partial \bar{\Psi}}{\partial \overline{\boldsymbol{F}}^{\text {iso }}}: \overline{\mathbb{I}}+\frac{\partial^{2} \bar{\Psi}}{\partial \overline{\boldsymbol{F}}^{\text {iso }} \partial \overline{\boldsymbol{F}}^{\text {iso }}}:\left[\overline{\mathbb{I}}-\frac{1}{2} \overline{\boldsymbol{F}} \otimes \overline{\boldsymbol{F}}^{-\mathrm{t}}\right]: \overline{\boldsymbol{F}}\right] .
\end{aligned}
$$

\section{Appendix 2: Differential geometry of two- dimensional manifolds embedded in three- dimensional space}

In this section we briefly review some common terminologies in differential geometry of two-dimensional manifolds frequently used in this work to represent the interface elasticity theory. Finding the shortest distance (minimal geodesic) on a curved two-dimensional manifold (interface) embedded in a three-dimensional Euclidean space is presented subsequently, which is employed in the calculation of the non-local coefficients on the interface.

A parametric interface $\mathscr{I}$ in $\mathbb{E}^{3}$ (three-dimensional embedding Euclidean space) is a map $\mathscr{I}: \mathcal{I} \rightarrow \mathbb{E}^{3}$ (with $\mathcal{I} \in \mathbb{E}^{2}$ ) such that its differential has rank 2 at all points $\eta^{\alpha} \in \mathcal{I}$ with $\alpha=1,2$. The interface can be defined by a parametric equation $\overline{\boldsymbol{x}}: \mathcal{I} \rightarrow \mathbb{E}^{3}$ as $\overline{\boldsymbol{x}}=$ $\overline{\boldsymbol{x}}\left(\eta^{\alpha}\right)$, where $\overline{\boldsymbol{x}}$ is a vector-valued function of the scalarvalued parameters $\eta^{\alpha}$. The tangent space to $\overline{\boldsymbol{x}}$ at $\eta^{\alpha}$ is 
(a)

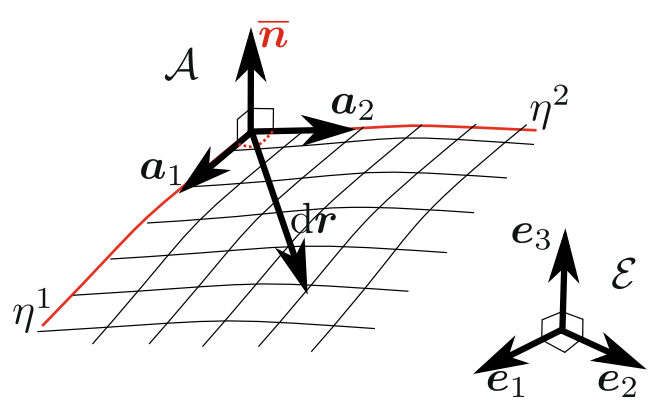

(b)

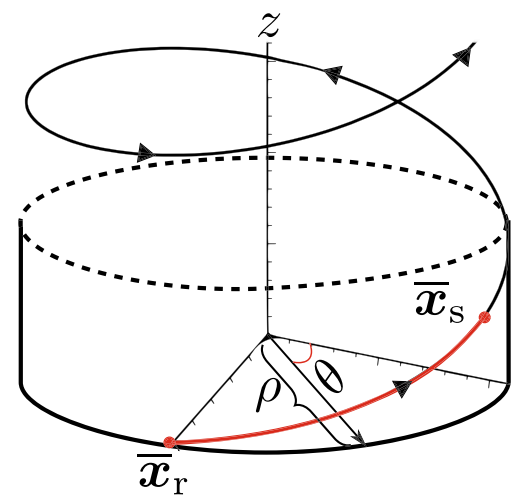

Fig. 16 Illustration of the Cartesian basis $\mathcal{E}$ and an arbitrary basis $\mathcal{A}$ (a), and a cylindrical interface with its minimal geodesic (helix) along with the polar coordinate system (b). The red portion of the helix on the cylinder is the shortest arc-length of all the curves connecting the point $\overline{\boldsymbol{x}}_{\mathrm{r}}$ and $\overline{\boldsymbol{x}}_{\mathrm{s}}$. In (a), the $\boldsymbol{a}_{1}$ and $\boldsymbol{a}_{2}$

the linear map $\mathrm{D} \overline{\boldsymbol{x}}\left(\eta^{\alpha}\right): \mathbb{E}^{2} \rightarrow \mathbb{E}^{3}$ denoted by $T \mathscr{I}$ where $\mathrm{D} \overline{\boldsymbol{x}}\left(\eta^{\alpha}\right)$ is the differential of the interface. The tangent vectors $\boldsymbol{a}_{\alpha} \in T \mathscr{I}$, i.e. the covariant interface basis vectors (see Fig. 16a) are then given by $\boldsymbol{a}_{\alpha}=$ $\partial_{\eta^{\alpha}} \overline{\boldsymbol{x}}\left(\eta^{\alpha}\right)$.

The corresponding contravariant (dual) interface basis vectors $\boldsymbol{a}^{\alpha}$ are related to the covariant interface basis vectors by means of the co- and contravariant interface metric coefficients $a_{\alpha \beta}$ (first fundamental form for the interface) and $a^{\alpha \beta}$, respectively, as

$\boldsymbol{a}^{\alpha}=a^{\alpha \beta} \boldsymbol{a}_{\beta} \quad$ and $\quad \boldsymbol{a}_{\alpha}=a_{\alpha \beta} \boldsymbol{a}^{\beta} \quad$ where
$a_{\alpha \beta}=\boldsymbol{a}_{\alpha} \cdot \boldsymbol{a}_{\beta} \quad$ and $\quad a^{\alpha \beta}=\boldsymbol{a}^{\alpha} \cdot \boldsymbol{a}^{\beta}$.

Note that the two metrics are inverse to each other, i.e. $\left[a^{\alpha \beta}\right]=\left[a_{\alpha \beta}\right]^{-1}$. The contra- and covariant base vectors $\boldsymbol{a}_{3}$ and $\boldsymbol{a}^{3}$, normal to $T \mathscr{I}$, are defined by

$\boldsymbol{a}^{3}:=\boldsymbol{a}_{1} \times \boldsymbol{a}_{2} \quad$ and $\quad \boldsymbol{a}_{3}:=\left[a^{33}\right]^{-1} \boldsymbol{a}^{3}$,

such that $\boldsymbol{a}^{3} \cdot \boldsymbol{a}_{3}=1$. Correspondingly the unit normal to the interface, parallel to $\boldsymbol{a}^{3}$ and $\boldsymbol{a}_{3}$, can be calculated as $\overline{\boldsymbol{n}}=\boldsymbol{a}_{3} /\left|\boldsymbol{a}_{3}\right|=\boldsymbol{a}^{3} /\left|\boldsymbol{a}^{3}\right|$. The interface identity tensor is defined as $\overline{\boldsymbol{i}}:=\boldsymbol{i}-\boldsymbol{a}_{3} \otimes \boldsymbol{a}^{3}=\boldsymbol{i}-\overline{\boldsymbol{n}} \otimes \overline{\boldsymbol{n}}$, where $\boldsymbol{i}$ denotes the ordinary mixed-variant unit tensor of the embedding Euclidean space. The interface gradient, divergence and determinant operators in a general curvilinear coordinate are defined as in $\mathcal{A}$ are not necessarily orthogonal to each other, whereas the Cartesian coordinate system $\mathcal{E}$ is composed of three orthogonal axes. In (b), every point on the surface of the cylinder is characterized by the coordinates $\rho$ the radius, $\theta$ the sweeping angle and $z$ the height

$\overline{\operatorname{grad}}\{\bar{\bullet}\}:=\frac{\partial\{\overline{\boldsymbol{\bullet}}\}}{\partial \eta^{\alpha}} \otimes \boldsymbol{a}_{\alpha}, \quad \overline{\operatorname{div}}\{\bar{\bullet}\}:=\frac{\partial\{\overline{\boldsymbol{\bullet}}\}}{\partial \eta^{\alpha}} \cdot \boldsymbol{a}_{\alpha} \quad$ and

$\overline{\operatorname{det}}\{\overline{\boldsymbol{\bullet}}\}:=\frac{\left[\{\overline{\boldsymbol{\bullet}}\} \cdot \boldsymbol{a}_{1}\right] \times\left[\{\overline{\boldsymbol{\bullet}}\} \cdot \boldsymbol{a}_{2}\right]}{\left|\boldsymbol{a}_{1} \times \boldsymbol{a}_{2}\right|}$.

Having obtained the normal to the interface, co- and contravariant basis vectors, the interface curvature tensor $\overline{\boldsymbol{k}}$, second-order superficial deformation gradient tensor $\overline{\boldsymbol{F}}$ and its inverse $\overline{\boldsymbol{f}}$ are defined, respectively, as

$\overline{\boldsymbol{k}}:=-\overline{\operatorname{grad}} \overline{\boldsymbol{n}}, \quad \overline{\boldsymbol{F}}:=\overline{\operatorname{Grad}} \overline{\boldsymbol{x}}=\boldsymbol{a}_{\alpha} \otimes \boldsymbol{A}^{\alpha} \quad$ and

$\overline{\boldsymbol{f}}:=\overline{\operatorname{grad}} \overline{\boldsymbol{X}}=\boldsymbol{A}_{\alpha} \otimes \boldsymbol{a}^{\alpha}$.

Note that due to the superficiality (rank deficiency) of the interface deformation gradient $\overline{\boldsymbol{F}}$, its inverse $\overline{\boldsymbol{f}}$ must be computed using Eq. (56) 3 .

The geodesics are the general form of straight lines when applied to curved, three-dimensional interfaces. The minimal geodesics in differential geometry are the shortest distance paths between two points on an interface. Clearly, minimal geodesics on the interfaces are curves of minimum arc-lengths. To find the minimal geodesics, first we introduce the parameter $t$ on which the interface parameters $\eta^{\alpha}$ are dependent. The arclength of the curve connecting any two points $\overline{\boldsymbol{x}}_{\mathrm{r}}\left(t_{1}\right)$ and $\overline{\boldsymbol{x}}_{\mathrm{S}}\left(t_{2}\right)$ on the curved interface can be written as

$I=\int_{t_{1}}^{t_{2}} \sqrt{a_{\alpha \beta} \frac{\mathrm{d} \eta^{\alpha}(t)}{\mathrm{d} t} \frac{\mathrm{d} \eta^{\beta}(t)}{\mathrm{d} t}} \mathrm{~d} t$ 
Table $6 L_{2}$ norm of the residual for three scenarios: bulk and interface damaged, only bulk damaged and only interface damaged

\begin{tabular}{|c|c|c|c|c|c|c|c|}
\hline Increment & Iteratio & & & & & & \\
\hline \multicolumn{8}{|c|}{ Bulk and interface damaged } \\
\hline 1 & 1.731 & $5.211 \mathrm{e}-07$ & & & & & \\
\hline 26 & 1.730 & $1.633 \mathrm{e}-02$ & $3.949 \mathrm{e}-04$ & $2.403 e-07$ & & & \\
\hline 28 & 1.730 & $4.195 e-02$ & $5.060 \mathrm{e}-02$ & $8.803 e-03$ & $2.187 \mathrm{e}-05$ & $8.620 \mathrm{e}-09$ & \\
\hline 99 & 1.725 & $1.919 \mathrm{e}-02$ & $2.322 \mathrm{e}-02$ & $7.821 \mathrm{e}-03$ & $6.502 \mathrm{e}-04$ & $1.385 \mathrm{e}-05$ & $2.211 \mathrm{e}-10$ \\
\hline 160 & 1.728 & $1.973 \mathrm{e}-02$ & $3.264 \mathrm{e}-03$ & $4.239 \mathrm{e}-04$ & $3.243 e-05$ & $3.385 \mathrm{e}-10$ & \\
\hline \multicolumn{8}{|c|}{ Only bulk damaged } \\
\hline 1 & 4.900 & $2.088 \mathrm{e}-06$ & $2.987 \mathrm{e}-10$ & & & & \\
\hline 41 & 4.886 & $2.152 \mathrm{e}-02$ & $1.627 \mathrm{e}-02$ & $1.331 \mathrm{e}-03$ & $8.096 \mathrm{e}-05$ & $9.492 \mathrm{e}-10$ & \\
\hline 89 & 4.867 & $1.669 \mathrm{e}-02$ & $2.073 \mathrm{e}-03$ & $8.317 \mathrm{e}-05$ & $2.649 \mathrm{e}-06$ & $2.641 \mathrm{e}-10$ & \\
\hline 134 & 4.894 & $1.053 \mathrm{e}-03$ & $1.781 \mathrm{e}-05$ & $3.111 \mathrm{e}-06$ & $3.130 \mathrm{e}-10$ & & \\
\hline 160 & 4.896 & $5.573 \mathrm{e}-04$ & $5.437 \mathrm{e}-06$ & $3.633 \mathrm{e}-10$ & & & \\
\hline \multicolumn{8}{|c|}{ Only interface damaged } \\
\hline 1 & 4.900 & $2.088 \mathrm{e}-06$ & $2.988 \mathrm{e}-10$ & & & & \\
\hline 14 & 4.894 & $4.816 \mathrm{e}-02$ & $1.142 \mathrm{e}-01$ & $1.339 \mathrm{e}-02$ & $1.982 \mathrm{e}-03$ & $4.944 \mathrm{e}-07$ & $3.949 \mathrm{e}-10$ \\
\hline 41 & 4.883 & $1.147 \mathrm{e}-02$ & $8.661 \mathrm{e}-06$ & $1.629 \mathrm{e}-09$ & & & \\
\hline 99 & 4.856 & $4.268 \mathrm{e}-03$ & $8.176 \mathrm{e}-07$ & & & & \\
\hline 160 & 4.829 & $1.146 \mathrm{e}-04$ & $7.868 \mathrm{e}-10$ & & & & \\
\hline
\end{tabular}

where the integrand is the infinitesimal line element. Next, to find the shortest arc-length of the curves connecting the two points (minimal geodesic), the functional $I$ is minimized. In doing so, the integrand in Eq. (57) (the Lagrangian), denoted by $L\left(\eta^{\alpha}(t), \dot{\eta}^{\alpha}(t)\right)$ must satisfy the Euler-Lagrange equations of the form

$$
\frac{\mathrm{d}}{\mathrm{d} t} \frac{\partial L}{\partial \dot{\eta}^{\alpha}}-\frac{\partial L}{\partial \eta^{\alpha}}=0
$$

where $\{\dot{\bullet}\}$ signifies a differentiation with respect to the parameter $t$.

\section{Convergence behavior and run-time analysis}

In this section we present some data on the convergence behavior and run-time of the computational problem at hand. Firstly, the $L_{2}$ norms of the residual of few increments for all the three examples discussed in Sect. 6 are given in Table 6. As mentioned before, due to the consistent linearization, the asymptotic quadratic rate of convergence associated with the Newton-Raphson scheme is achieved. Secondly, to study how the interactive radius would change the memory consumption and run-time, we only allow the damage initiation in the bulk, increase both number of elements ${ }^{7}$ and interactive radius $R$ and measure the run-time for one iteration per increment. These measurements are carried out on a machine with the following specifications:

- processors: Intel Core i7-4770 CPU $3.40 \mathrm{GHz} \times 8$,

- memory: 15.6GB,

- OS type: 64-bit.

The run-time measurements per iteration together with the memory usage for a serial and parallel code are given in Table 7. The parallel implementation is carried out using the MPI library. Note that the total run-time for a mesh size of $100 \times 100 \times 1$ with $R=0.01 \mathrm{~mm}$ is approximately $39.3 \mathrm{~h}$. A parallel implementation, using 4 processors, results in a speed-up of 3.5 and consequently a run-time of $7.75 \mathrm{~h}$. As indicated by the presented data in Table 7, increasing the interactive radius $R$ causes a substantial increase in the run-time. The main source of the time consumption, as expected, is in the stiffness assembly since firstly the non-localization is integral-type and secondly the linearization is consistent (introducing the double integrals in the stiffness

\footnotetext{
$\overline{7 \text { In Table } 7,10} \times 10 \times 1$ for instance indicates 10 elements in $x, y$ and 1 element in $z$ direction.
} 
Table 7 Serial- and parallel-code run-time measurements per iteration together with the memory consumption for various mesh sizes and values of interactive radius

\begin{tabular}{|c|c|c|c|}
\hline \multicolumn{4}{|c|}{ serial } \\
\hline & local & \multicolumn{2}{|c|}{ nonlocal } \\
\hline $10 \times 10 \times 1$ & $R=0 \mathrm{~mm}$ & $R=0.01 \mathrm{~mm}$ & $R=0.02 \mathrm{~mm}$ \\
\hline $\begin{array}{l}\text { time } \\
\text { memory }\end{array}$ & $\begin{array}{c}3.39 \mathrm{~s} \\
5.1 \mathrm{MB}\end{array}$ & $\begin{array}{c}4.2 \mathrm{~s} \\
5.3 \mathrm{MB}\end{array}$ & $\begin{array}{c}7.24 \mathrm{~s} \\
5.3 \mathrm{MB}\end{array}$ \\
\hline $40 \times 40 \times 1$ & $R=0 \mathrm{~mm}$ & $R=0.01 \mathrm{~mm}$ & $R=0.02 \mathrm{~mm}$ \\
\hline $\begin{array}{l}\text { time } \\
\text { memory }\end{array}$ & $\begin{array}{c}25.35 \mathrm{~s} \\
31.1 \mathrm{MB}\end{array}$ & $\begin{array}{c}69.65 \mathrm{~s} \\
59.3 \mathrm{MB}\end{array}$ & $\begin{array}{c}129.5 \mathrm{~s} \\
63.7 \mathrm{MB}\end{array}$ \\
\hline $100 \times 100 \times 1$ & $R=0 \mathrm{~mm}$ & $R=0.01 \mathrm{~mm}$ & $R=0.02 \mathrm{~mm}$ \\
\hline $\begin{array}{l}\text { time } \\
\text { total time* } \\
\text { memory }\end{array}$ & $\begin{array}{l}68.95 \mathrm{~s} \\
144 \mathrm{MB}\end{array}$ & $\begin{array}{c}122 \mathrm{~s} \\
27.11 \mathrm{hr} \\
582 \mathrm{MB}\end{array}$ & $\begin{array}{c}177 \mathrm{~s} \\
39.33 \mathrm{hr} \\
583 \mathrm{MB}\end{array}$ \\
\hline \multicolumn{4}{|c|}{ parallel } \\
\hline & local & \multicolumn{2}{|c|}{ nonlocal } \\
\hline $10 \times 10 \times 1$ & $R=0 \mathrm{~mm}$ & $R=0.01 \mathrm{~mm}$ & $R=0.02 \mathrm{~mm}$ \\
\hline $\begin{array}{l}\text { time } \\
\text { memory }\end{array}$ & $\begin{array}{c}0.97 \mathrm{~s} \\
20.4 \mathrm{MB}\end{array}$ & $\begin{array}{c}1.20 \mathrm{~s} \\
26.2 \mathrm{MB}\end{array}$ & $\begin{array}{c}2.04 \mathrm{~s} \\
26 \mathrm{MB}\end{array}$ \\
\hline $40 \times 40 \times 1$ & $R=0 \mathrm{~mm}$ & $R=0.01 \mathrm{~mm}$ & $R=0.02 \mathrm{~mm}$ \\
\hline $\begin{array}{l}\text { time } \\
\text { memory }\end{array}$ & $\begin{array}{c}7.24 \mathrm{~s} \\
120.9 \mathrm{MB}\end{array}$ & $\begin{array}{c}19.9 \mathrm{~s} \\
230.1 \mathrm{MB}\end{array}$ & $\begin{array}{c}57 \mathrm{~s} \\
245.7 \mathrm{MB}\end{array}$ \\
\hline $100 \times 100 \times 1$ & $R=0 \mathrm{~mm}$ & $R=0.01 \mathrm{~mm}$ & $R=0.02 \mathrm{~mm}$ \\
\hline $\begin{array}{l}\text { time } \\
\text { total time* } \\
\text { memory }\end{array}$ & $\begin{array}{c}19.7 \mathrm{~s} \\
561.6 \mathrm{MB}\end{array}$ & $\begin{array}{c}34.9 \mathrm{~s} \\
7.75 \mathrm{hr} \\
2269 \mathrm{MB}\end{array}$ & $\begin{array}{c}79.2 \mathrm{~s} \\
17.6 \mathrm{hr} \\
2273 \mathrm{MB}\end{array}$ \\
\hline
\end{tabular}

formulation). However, as mentioned in Jirásek and Patzák (2002), if this type of non-localization is chosen, the extra time spent on the stiffness assembly due to consistent linearization in every iteration might be compensated by fewer iterations required to meet the convergence criterion. This matter becomes critical if a more stringent convergence criterion is necessary. For further details on the implementation issues of the consistent linearization of non-local damage problems of integral-type we refer to Jirásek and Patzák (2002).

\section{References}

Alfano G, Crisfield MA (2001) Finite element interface models for the delamination analysis of laminated composites: Mechanical and computational issues. Int J Numer Methods Eng 50:1701-1736

Alfano G, Sacco E (2006) Combining interface damage and friction in a cohesive-zone model. Int J Numer Methods Eng 68(5):542-582

Allix O, Ladevéze P, Corigliano A (1995) Damage analysis of interlaminar fracture specimens. Compos Struct 31(1):6174

Allix O, Corigliano A (1996) Modeling and simulation of crack propagation in mixed-modes interlaminar fracture specimens. Int J Fract 77(2):111-140
Andrade FXC, Sá JMACD, Pires FMA (2011) A ductile damage nonlocal model of integral-type at finite strains: formulation and numerical issues. Int J Damage Mech 20(4):515-557

Andrade F, Sá JCD, Pires FA (2014) Assessment and comparison of non-local integral models for ductile damage. Int $\mathrm{J}$ Damage Mech 23(2):261-296

Aragón AM, Soghrati S, Geubelle PH (2013) Effect of in-plane deformation on the cohesive failure of heterogeneous adhesives. J Mech Phys Solids 61(7):1600-1611

Askes H, Sluys LJ (2000) Remeshing strategies for adaptive ALE analysis of strain localisation. Eur J Mech A Solids 19(3):447-467

Barenblatt GI (1962) The mathematical theory of equilibrium cracks in brittle fracture. Advances in applied mechanics 7(1):55-129

Bažant ZP (1994) Nonlocal damage theory based on micromechanics of crack interactions. J Eng Mech 120(3):593-617

Bažant ZP, Cabot GP (1989) Measurement of characteristic length of nonlocal continuum. J Eng Mech 115(4):755-767

Bažant ZP, Jirásek M (2002) Nonlocal integral formulations of plasticity and damage: survey of progress. J Eng Mech 128(11):1119-1149

Bažant ZP, Xi Y (1991) Statistical size effect in quasibrittle structures: II. Nonlocal theory. J Eng Mech 117(11):2623-2640

Benveniste Y (2006) A general interface model for a threedimensional curved thin anisotropic interphase between two anisotropic media. J Mech Phys Solids 54(4):708-734

Benveniste Y (2013) Models of thin interphases and the effective medium approximation in composite media with curvilinearly anisotropic coated inclusions. Int J Eng Sci 72:140 154

Benveniste Y, Miloh T (2001) Imperfect soft and stiff interfaces in two-dimensional elasticity. Mech Mater 33(6):309-323

Bolzon G, Corigliano A (1997) A discrete formulation for elastic solids with damaging interfaces. Comput Methods Appl Mech Eng 140(3-4):329-359

Cabot GP, Bažant ZP (1987) Nonlocal damage theory. J Eng Mech 113(10):1512-1533

Cammarata RC (1997) Surface and interface stress effects on interfacial and nanostructured materials. Mater Sci Eng A 237(2):180-184

Carmeliet J (1999) Optimal estimation of gradient damage parameters from localization phenomena in quasi-brittle materials. Mech Cohes Frict Mater 4(1):1-16

Cazes F, Coret M, Combescure A, Gravouil A (2009) A thermodynamic method for the construction of a cohesive law from a nonlocal damage model. Int J Solids Struct 46(6):14761490

Chaboche JL (1981) Continuous damage mechanics-a tool to describe phenomena before crack initiation. Nucl Eng Des 64(2):233-247

Chaboche JL (1984) Anisotropic creep damage in the framework of continuum damage mechanics. Nucl Eng Des 79(3):309_ 319

Chaboche JL, Girard R, Schaff A (1997) Numerical analysis of composite systems by using interphase/interface models. Comput Mech 20:3-11

Chatzigeorgiou G, Javili A, Steinmann P (2013) Multiscale modelling for composites with energetic interfaces at the microor nanoscale. Math Mech Solids 
Chen J, Crisfield M, Kinloch AJ, Busso EP, Matthews FL, Qiu Y (1999) Predicting progressive delamination of composite material specimens via interface elements. Mech Compos Mater Struct 6(4):301-317

Cordero NM, Forest S, Busso EP (2015) Second strain gradient elasticity of nano-objects. J Mech Phys Solids. doi:10.1016/ j.jmps.2015.07.012

Corigliano A (1993) Formulation, identification and use of interface models in the numerical analysis of composite delamination. Int J Solids Struct 30(20):2779-2811

Daher N, Maugin GA (1986) The method of virtual power in continuum mechanics application to media presenting singular surfaces and interfaces. Acta Mech 60(3-4):217-240

Davydov D, Javili A, Steinmann P (2013) On molecular statics and surface-enhanced continuum modeling of nanostructures. Comput Mater Sci 69:510-519

de Souza Neto EA, Perić D, Owen DRJ (1994a) A model for elastoplastic damage at finite strains: algorithmic issues and applications. Eng Comput 11(3):257-281

de Souza Neto EA, Perić D, Owen DRJ (1994b) A phenomenological three-dimensional rate-idependent continuum damage model for highly filled polymers: formulation and computational aspects. J Mech Phys Solids 42(10):1533-1550

de Souza Neto EA, Perić D, Owen DRJ (1998) Continuum modelling and numerical simulation of material damage at finite strains. Arch Comput Methods Eng 5(4):311-384

de Souza Neto EA, Peric D, Owen DRJ (2011) Computational methods for plasticity: theory and applications. Wiley, London

de Souza Neto EA, Perić D (1996) A computational framework for a class of fully coupled models for elastoplastic damage at finite strains with reference to the linearization aspects. Comput Methods Appl Mech Eng 130(1-2):179-193

dell'Isola F, Romano A (1987) On the derivation of thermomechanical balance equations for continuous systems with a nonmaterial interface. Int J Eng Sci 25:1459-1468

Dingreville R, Qu J, Cherkaoui M (2005) Surface free energy and its effect on the elastic behavior of nano-sized particles, wires and films. J Mech Phys Solids 53(8):1827-1854

Duan HL, Karihaloo BL (2007) Effective thermal conductivities of heterogeneous media containing multiple imperfectly bonded inclusions. Phys Rev B 75(6):064,206

Duan HL, Wang J, Huang ZP, Karihaloo BL (2005a) Eshelby formalism for nano-inhomogeneities. Proc R Soc A Math Phys Eng Sci 461(2062):3335-3353

Duan HL, Wang J, Huang ZP, Karihaloo BL (2005b) Sizedependent effective elastic constants of solids containing nano-inhomogeneities with interface stress. J Mech Phys Solids 53(7):1574-1596

Duan HL, Wang J, Karihaloo BL (2009) Theory of elasticity at the nanoscale. Adv Appl Mech 42:1-68

Eringen AC (1966) A unified theory of thermomechanical materials. Int J Eng Sci 4(2):179-202

Fagerström M, Larsson R (2008) A thermo-mechanical cohesive zone formulation for ductile fracture. J Mech Phys Solids 56(10):3037-3058

Fischer FD, Waitz T, Vollath D, Simha NK (2008) On the role of surface energy and surface stress in phase-transforming nanoparticles. Prog Mater Sci 53(3):481-527
Fischer FD, Svoboda J (2010) Stresses in hollow nanoparticles. Int J Solids Struct 47(20):2799-2805

Fleischhauer R, Behnke R, Kaliske M (2013) A thermomechanical interface element formulation for finite deformations. Comput Mech 52(5):1039-1058

Fried E, Todres R (2005) Mind the gap: the shape of the free surface of a rubber-like material in proximity to a rigid contactor. J Elast 80(1-3):97-151

Gurtin ME, Weissmüller J, Larché F (1998) A general theory of curved deformable interfaces in solids at equilibrium. Philos Mag A 78(5):1093-1109

Gurtin ME, Murdoch AI (1975) A continuum theory of elastic material surfaces. Arch Ration Mech Anal 57(4):291-323

Haiss W (2001) Surface stress of clean and adsorbate-covered solids. Rep Prog Phys 64(5):591

Holzapfel GA (2000) Nonlinear Solid mechanics: a continuum approach for engineering. Wiley, London

Huang ZP, Sun L (2007) Size-dependent effective properties of a heterogeneous material with interface energy effect: From finite deformation theory to infinitesimal strain analysis. Acta Mech 190(1-4):151-163

Ijaz H, Asad M, Gornet L, Alam SY (2014) Prediction of delamination crack growth in carbon/fiber epoxy composite laminates using non-local interface damage model. Mech Ind 15(4):293-300

Javili A, McBride A, Steinmann P (2012) Numerical modelling of thermomechanical solids with mechanically energetic (generalised) Kapitza interfaces. Comput Mater Sci 65:542-551

Javili A, dell'Isola F, Steinmann P (2013a) Geometrically nonlinear higher-gradient elasticity with energetic boundaries. J Mech Phys Solids 61(12):2381-2401

Javili A, McBride A, Steinmann P (2013b) Numerical modelling of thermomechanical solids with highly conductive energetic interfaces. Int J Numer Meth Eng 93(5):551-574

Javili A, McBride A, Steinmann P (2013c) Thermomechanics of solids with lower-dimensional energetics: on the importance of surface, interface, and curve structures at the nanoscale. A unifying review. Appl Mech Rev 65(1):010802

Javili A, Kaessmair S, Steinmann P (2014a) General imperfect interfaces. Comput Methods Appl Mech Eng 275:76-97

Javili A, McBride A, Steinmann P, Reddy BD (2014b) A unified computational framework for bulk and surface elasticity theory: a curvilinear-coordinate-based finite element methodology. Comput Mech 54(3):745-762

Javili A, Steinmann P (2009) A finite element framework for continua with boundary energies. Part I: the two-dimensional case. Comput Methods Appl Mech Eng 198(27-29):21982208

Javili A, Steinmann P (2010a) A finite element framework for continua with boundary energies. Part II: the threedimensional case. Comput Methods Appl Mech Eng 199(912):755-765

Javili A, Steinmann P (2010b) On thermomechanical solids with boundary structures. Int J Solids Struct 47(24):3245-3253

Jirasek M (1998) Nonlocal models for damage and fracture: comparison of approaches. Int J Solids Struct 35(31):4133-4145

Jirásek M, Patzák B (2002) Consistent tangent stiffness for nonlocal damage models. Comput Struct 80(14-15):1279-1293 
Kachanov LM (1958) Time of the rupture process under creep conditions. Izv Akad Nauk SSR Otd Tech Nauk 8:26-31

Krajcinovic D, Fonseka GU (1981) Continuous damage theory of brittle materials- 1 . General theory. J Appl Mech Trans ASME 48(4):809-815

Krayani A, Pijaudier-Cabot G, Dufour F (2009) Boundary effect on weight function in nonlocal damage model. Eng Fract Mech 76(14):2217-2231

Ladevèze P, Allix O, Gornet L, Lévêque D, Perret L (1998) A computational damage mechanics approach for laminates: identification and comparison with experimental results. Stud Appl Mech 46:481-500

Lemaitre J (1984) Three-dimensional ductile damage model applied to deep-drawing forming limits. In: Proceedings of the $4^{\text {th }}$ international conference on the mechanical behaviour of materials vol 2, pp 1047-1053

Levitas VI, Javanbakht M (2010) Surface tension and energy in multivariant martensitic transformations: phase-field theory, simulations, and model of coherent interface. Phys Rev Lett 105(16):165-701

Lin G, Geubelle PH, Sottos NR (2001) Simulation of fiber debonding with friction in a model composite pushout test. Int J Solids Struct 38(46-47):8547-8562

Mazars J, Berthaud Y, Ramtani S (1990) The unilateral behaviour of damaged concrete. Eng Fract Mech 35(4):629-635

McBride AT, Javili A, Steinmann P, Bargmann S (2011) Geometrically nonlinear continuum thermomechanics with surface energies coupled to diffusion. J Mech Phys Solids 59(10):2116-2133

Mi Y, Crisfield MA, Davies GAO, Hellweg HB (1998) Progressive delamination using interface elements. J Compos Mater 32(14):1246-1272

Moeckel GP (1975) Thermodynamics of an interface. Arch Ration Mech Anal 57(3):255-280

Mosler J, Scheider I (2011) A thermodynamically and variationally consistent class of damage-type cohesive models. J Mech Phys Solids 59(8):1647-1668

Murakami S, Ohno N (1981) A continuum theory of creep and creep damage. In: Ponter ARS, Hayhurst DR (eds) Creep in structures, international union of theoretical and applied mechanics. Springer, Berlin, pp 422-444

Murdoch AI (1976) A thermodynamical theory of elastic material interfaces. Q J Mech Appl Math 29(3):245-275

Needleman A (1990) An analysis of tensile decohesion along an interface. J Mech Phys Solids 38(3):289-324

Needleman A (1992) Micromechanical modelling of interfacial decohesion. Ultramicroscopy 40:203-214

Needleman A (2014) Some issues in cohesive surface modeling. Procedia IUTAM 10:221-246

Özdemir I, Brekelmans WAM, Geers MGD (2010) A thermomechanical cohesive zone model. Comput Mech 46:735745

Park HS, Klein PA (2007) Surface Cauchy-Born analysis of surface stress effects on metallic nanowires. Phys Rev B 75(085):408

Parrinello F, Failla B, Borino G (2009) Cohesive-frictional interface constitutive model. Int J Solids Struct 46(13):26802692
Pijaudier-Cabot G, Dufour F (2010) Non local damage model: boundary and evolving boundary effects. Eur J Environ Civil Eng 14(6-7):729-749

Pijaudier-Cabot G, Grégoire D (2014) A review of non local continuum damage: modelling of failure? Netw Heterog Media 9(4):575-597

Pijaudier-Cabot G, Huerta A (1991) Finite element analysis of bifurcation in nonlocal strain softening solids. Comput Methods Appl Mech Eng 90(1-3):905-919

Rabotnov YN (1963) On the equation of state of creep. Proc Inst Mech Eng Conf Proc 178(1):2-117-2-122

Raous M (2011) Interface models coupling adhesion and friction. Comptes Rendus Mecanique 339(7-8):491-501

Saanouni K, Chaboche JL, Lesne PM (1989) On the creep crackgrowth prediction by a non local damage formulation. Eur J Mech A Solids 8(6):437-459

Saouridis C, Mazars J (1992) Prediction of the failure and size effect in concrete via a bi-scale damage approach. Eng Comput 9(3):329-344

Schellekens JCJ, Borst RD (1993) A non-linear finite element approach for the analysis of mode-I free edge delamination in composites. Int J Solids Struct 30(9):1239-1253

Sharma P, Ganti S, Bhate N (2003) Effect of surfaces on the size-dependent elastic state of nano-inhomogeneities. Appl Phys Lett 82(4):535-537

Sharma P, Ganti S (2004) Size-dependent Eshelbys tensor for embedded nano-inclusions incorporating surface/interface energies. J Appl Mech 71(5):663-671

Sharma P, Wheeler LT (2007) Size-dependent elastic state of ellipsoidal nano-inclusions incorporating surface/ interface tension. J Appl Mech 74(3):447-454

Simo JC, Hughes TJR (1998) Comput Inelast. Springer, New York

Simo JC, Ju JW (1987) Strain- and stress-based continuum damage models I: formulation. Int J Solids Struct 23(7):821-840

Steigmann DJ, Ogden RW (1999) Elastic surface-substrate interactions. Proc R Soc Lond A Math Phys Eng Sci 455(1982):437-474

Steinmann P, Miehe C, Stein E (1994) Comparison of different finite deformation inelastic damage models within multiplicative elastoplasticity for ductile materials. Comput Mech 13(6):458-474

Steinmann P (1999) Formulation and computation of geometrically non-linear gradient damage. Int J Numer Meth Eng 46(5):757-779

Steinmann P (2008) On boundary potential energies in deformational and configurational mechanics. J Mech Phys Solids 56(3):772-800

Tvergaard V (1990) Effect of fibre debonding in a whiskerreinforced metal. Mater Sci Eng A 125(2):203-213

van den Bosch MJ, Schreurs PJG, Geers MGD (2006) An improved description of the exponential $\mathrm{Xu}$ and Needleman cohesive zone law for mixed-mode decohesion. Eng Fract Mech 73:1220-1234

van den Bosch MJ, Schreurs PJG, Geers MGD (2007) A cohesive zone model with a large displacement formulation accounting for interfacial fibrilation. Eur J Mech A/Solids 26:1-19 
Willam K, Rhee I, Shing B (2004) Interface damage model for thermomechanical degradation of heterogeneous materials. Comput Methods Appl Mech Eng 193(30):3327-3350

Wu L, Becker G, Noels L (2014) Elastic damage to crack transition in a coupled non-local implicit discontinuous Galerkin/extrinsic cohesive law framework. Comput Methods Appl Mech Eng 279:379-409

Yvonnet J, He QC, Zhu QZ, Shao JF (2011a) A general and efficient computational procedure for modelling the Kapitza thermal resistance based on XFEM. Comput Mater Sci 50(4):1220-1224
Yvonnet J, Mitrushchenkov A, Chambaud G, He QC (2011b) Finite element model of ionic nanowires with sizedependent mechanical properties determined by ab initio calculations. Comput Methods Appl Mech Eng 200(58):614-625 